\title{
Entwicklung bodengebundener Notarzteinsätze im Stadtgebiet Leipzig von 2003 bis 2013
}

\author{
Dissertation \\ zur Erlangung des akademischen Grades \\ Dr. med. \\ an der Medizinischen Fakultät \\ der Universität Leipzig
}

Eingereicht von

Katja Bader, geb. Jung

geboren am 19. Juni 1985 in Leipzig

Angefertigt an / in

Zentrale Notaufnahme, Universitätsklinikum Leipzig AöR

Betreut von

Dr. med. Alexandra Ramshorn-Zimmer

Privatdozent Dr. med. habil. Michael Bernhard

Professor Dr. med. André Gries

Februar 2018

Beschluss über die Verleihung des Doktorgrads vom: 20.11.2018 


\section{Inhaltsverzeichnis}

Bibliografische Beschreibung $\quad 2$

$\begin{array}{ll}\text { Abkürzungsverzeichnis } & 3\end{array}$

1. Einführung 4

1.1. Geschichtliche Aspekte der Notfallrettung in Deutschland 4

1.2. Aktuelles Rettungsdienstsystem 9

1.3. Ableitung der Studie / Wissenschaftlicher Hintergrund 10

$\begin{array}{ll}\text { 1.4. Publikation der Studie } & 13\end{array}$

$\begin{array}{ll}\text { 2. Publikation } & 14\end{array}$

3. Zusammenfassung 25

4. Literaturverzeichnis 29

5. Selbstständigkeitserklärung 33

6. Erklärung über den Eigenanteil an der vorliegenden Arbeit 34

$\begin{array}{ll}\text { 7. Lebenslauf } & 35\end{array}$

8. Vorstellung auf wissenschaftlichen Kongressen 37

9. Danksagung 38 


\section{Bibliographische Beschreibung}

Bader, Katja

\section{Entwicklung bodengebundener Notarzteinsätze im Stadtgebiet Leipzig von 2003 bis 2013}

Universität Leipzig, Dissertation

38 S., 45 Lit.

\section{Referat:}

Im deutschen Rettungsdienst wird über die kontinuierlich steigende Zahl von Notarzteinsätzen berichtet. Bisher lagen jedoch kaum Daten aus Großstädten vor, die diese Veränderungen über einen längeren Zeitraum darstellen.

In einer retrospektiven Untersuchung wurden die Notarzteinsätze jeweils des Zeitraums 01. Januar bis 31. März der Jahre 2003 und 2013 der Stadt Leipzig ausgewertet. Neben der Notarzteinsatzrate (Notarzteinsätze/1000 Einwohner und Jahr) wurden die Erkrankungs- und Verletzungsschwere (National Advisory Committee of Aeronautics [NACA]-Score), der Einsatzort und der Versorgungsgrad der Zielklink erfasst.

Die Zahl der Notarzteinsätze in den Beobachtungszeiträumen nahm zwischen 2003 und 2013 um $24 \%$ ( $n=6030$ vs. $n=7470$ ) und die Notarzteinsatzrate um $21 \%$ (48 vs. 58 ) zu. Bei steigendem Patientenalter (66 vs. 70 Jahre) stieg der Anteil hochbetagter [Alter $\geq 85$ Jahre: $11 \%$ vs. $16 \%(p<0,01)$ und der Anteil vital gefährdeter Patienten (NACA IV-VI: $14 \%$ vs. 16\% $(p<0,01)$ ]. Notarzteinsätze in Pflegeheimen vervierfachten sich [ $n=175$ (3\%) vs. $n=750$ (10\%), $p<0,01$ ] und die Zahl der Krankenhauseinweisungen stieg an [ $=3049$ (51 \%) vs. $n=$ 4738 (66\%), $p<0,01]$. Hierbei nahm maßgeblich der Anteil den Maximalversorgern zugeführter Patienten zu [ $n=1742(29 \%)$ vs. $n=3436(46 \%), p<0,01]$.

Im Stadtgebiet Leipzig wurden zwischen 2003 und 2013 steigende Notarzteinsatzzahlen und ein Anstieg schwer erkrankter und geriatrischer Patienten nachgewiesen. Die im Vergleich zum Bundesdurchschnitt nahezu doppelt so hohe Notarzteinsatzrate kann jedoch auf Defizite im Bereich der ambulanten Grundversorgung und Optimierungsmöglichkeiten bei der Disposition der Rettungsleitstelle hinweisen. 


\section{Abkürzungsverzeichnis}

\begin{tabular}{|c|c|}
\hline ADAC & Allgemeiner Deutscher Automobilclub \\
\hline Abb. & Abbildung \\
\hline DDR & Deutsche Demokratische Republik \\
\hline DIVI & Deutsche Interdisziplinäre Vereinigung für Intensiv- und Notfallmedizin \\
\hline DIVIDOK & $\begin{array}{l}\text { Deutsche Interdisziplinäre Vereinigung für Intensiv- und Notfallmedizin } \\
\text { Dokument }\end{array}$ \\
\hline DRK & Deutsches Rotes Kreuz \\
\hline EDV & Elektronische Datenverarbeitung \\
\hline EKG & Elektrokardiogramm \\
\hline evtl. & eventuell \\
\hline GCS & Glasgow Coma Skala \\
\hline ggf. & gegebenenfalls \\
\hline MEES & Mainzer Emergency Evaluation Score \\
\hline MIND & Minimaler Notfalldatensatz \\
\hline MW & Mittelwert \\
\hline NACA & National Advisory Commitee of Aeronautics \\
\hline NAW & Notarztwagen \\
\hline NEF & Notarzteinsatzfahrzeug \\
\hline $\mathrm{OP}$ & Operation \\
\hline RTW & Rettungswagen \\
\hline SD & Standardabweichung \\
\hline SPSS & Statistical Package for the Social Sciences \\
\hline Tab. & Tabelle \\
\hline vs. & versus \\
\hline z.B. & zum Beispiel \\
\hline z.T. & zum Teil \\
\hline
\end{tabular}




\section{Einführung}

\subsection{Geschichtliche Aspekte der Notfallrettung in Deutschland}

Die Versorgung von Notfällen beschränkte sich zunächst auf den militärischen Bereich, wobei es sich lediglich um Hilfeleistungen für Verwundete handelte. Ab dem 18. Jahrhundert begann sich die Notfallversorgung auch im zivilen Bereich zu etablieren. Diese gründete sich im Wesentlichen auf private, vorwiegend kirchliche und caritative Initiativen, wobei auch hier die Versorgung von Verletzten im Vordergrund stand [22]. Ein der Behandlung vor Ort folgender Transport zu einem Arzt oder einer Krankenstationen war nicht garantiert. Die damals tätigen Organisationen handelten dabei ohne gesetzliche Rahmenbedingungen, so dass kaum von einem systematisch organisierten Rettungsdienst die Rede sein konnte.

Der wahrscheinlich erste Notarzt war Adalbert Vinzenz Zarda. Am 04.11.1792 hielt er an der Ferdinandeischen Universität zu Prag seine erste Vorlesung über die „Rettungsmittel in plötzlicher Lebensgefahr". Seine Vorlesung beschloss er mit den Worten:

"Auch erbiete ich mich hiermit zu jedem der verzeichneten Unglücksfälle zu eilen und meine Kräfte anzuwenden, eine dem Tode schon Geopferten der Zahl der Lebenden wieder zurückzubringen, wenn mich diejenigen, welche als erste zugegen sind, davon verständigen und in meiner Wohnung in der Altstätter Bergmannsgasse wollen rufen lassen." [6]

Sicher waren hier die Einhaltung von Hilfsfristen und die konkreten therapeutischen Optionen begrenzt, dennoch zeichnen sich mit dieser Äußerung erste Grundgedanken unseres heutigen Notfallrettungssystems ab.

Neben der Behandlung der Verwundeten begann mit dem ausklingenden 18. Jahrhundert auch der Transport der Verletzten, der - von „Krankenträgern“ übernommen - den Beginn der ersten organisierten Rettungsdienste darstellte. Diese wurden im öffentlichen Bereich angesiedelt und teils von Feuerwehren oder (freiwilligen) Rettungsdiensten übernommen. Nach und nach wurden nationale Gesellschaften gegründet, so auch das Rote Kreuz 1863, 
der Arbeiter Samariter Bund (ASB) 1909, der an die katholische Kirche angebundene Malteser Hilfsdienst und die Johanniter Unfallhilfe der evangelischen Kirchen.

Bereits 1908 fand der erste Internationale Rettungskongress in Frankfurt statt und erste Forderungen nach einer ärztlichen Notfallversorgung vor Ort wurden laut. Der Rettungsdienst wurde, ähnlich den Feuerwehren und Polizei, zunehmend als öffentliche Aufgabe betrachtet. Im Rahmen des Kongresses erarbeiteten Wissenschaftler aller beteiligten Fachgebiete die Grundforderungen für eine „präklinische Notfallversorgung“. [38]

Kernthesen der abschließenden Verlautbarung waren:

- Es ist dafür Sorge zu tragen, dass zu jeder Zeit und überall erfahrene ärztliche Hilfe für Jedermann schnell verfügbar wird.

- Die Organisation der Erstversorgung, der Einsatz des Personals und der Fahrzeuge müssen in ärztlichen Händen liegen.

- In den Krankenhäusern sind geeignete Örtlichkeiten für die Erstversorgung von Notfällen zu schaffen, qualifizierte Ärzte dafür vor zu halten.

- Die Organisation der Rettungsdienste ist auf Massenunfälle auszurichten und dabei eine wirksame ärztliche Leitung sicher zu stellen.

- Es gibt kein medizinisches Fachgebiet, das alle Aufgaben im Rettungsdienst abdeckt. Alle müssen voneinander lernen. Eine dreimonatige Volontärzeit ist für jeden Arzt als Ausbildung zu fordern und schließlich ist der Rettungsdienst eine unabweisbare öffentliche staatliche Aufgabe der Vor- und Fürsorge.

Somit wurden bereits vor über 100 Jahren die Kerngedanken und -aufgaben unseres heutigen Notfallrettungssystems öffentlich formuliert bzw. angemahnt. Die Entwicklung zu einem funktionstüchtigen System, welches bestmöglich jene Forderungen zu erfüllen versucht, dauerte jedoch noch mehrere Jahrzehnte an.

Nach der Zeit der Weltkriege stand zunächst die Versorgung von Verkehrsunfallopfern im Mittelpunkt. Dabei fand die Methode des „scoop and run“ Anwendung, bei der der Patient auf dem schnellsten Weg, ohne medizinische Versorgungsmaßnahmen vor Ort, in das nächstgelegene Krankenhaus transportiert wurde. Änderungsbestrebungen dieses rein auf den Krankentransport ausgerichteten Systems gab es vielerorts, so beschreibt der Leipziger 
Kassenarzt Dr. Bruno Gittner in seinen handschriftlichen Lebenserinnerungen von 1936 beispielsweise: „Im Falle von lebensbedrohlichen Notfällen, die über den Polizeinotruf gemeldet wurden, schickte die Polizeihauptwache in der Wächterstrasse einen Streifenwagen mit Martinshorn und Blaulicht und nahm den diensthabenden Sprengelarzt vor dessen Praxis oder Wohnung auf und transportierte ihn zum Patienten." [15]

Darüber hinaus berichtete er, dass man sich schon zum damaligen Zeitpunkt Gedanken über die spezielle Sanitätsausbildung von Polizeibeamten machte.

Maßgeblich für die weitere Entwicklung der präklinischen Notfallversorgung waren die Thesen des Heidelberger Chirurgen Martin Kirschner in seinem richtungsweisenden Vortrag „Der Verkehrsunfall und seine erste Behandlung“, den er im Jahre 1938 in Berlin vor der 62. Tagung der Deutschen Gesellschaft für Chirurgie hielt. Dabei postulierte er zum einen

„„,....Wir müssen soweit kommen, dass nicht mehr der Schwerverletzte zum Arzt, sondern der Arzt zum Schwerverletzten gebracht wird"“"

und

„...lieber ein gesicherter Transport über 100 Kilometer in eine geeignete Klinik, als der schnelle Transport über die Straße in eine ungeeignete Arztpraxis. " [26]

Mit diesen Prämissen hatte Kirschner eine grundlegende Neuorientierung der präklinischen Versorgung hin zu einem arztgestützten Rettungssystem angeregt und auch erstmals die Bedeutung des Transportzieles für die weitere klinische Behandlung hervorgehoben. Hintergrund dieser Forderung war, dass zur damaligen Zeit 90\% der Unfalltoten am Unfallort, auf dem Weg zum Krankenhaus bzw. noch innerhalb der ersten 24 Stunden nach dem Unfallereignis verstarben. Kirschners Forderung nach Aufbau eines flächendeckenden, arztgestützten Rettungswesens wurde allerdings erst mit Beginn der 1970er Jahre umgesetzt.

Zuvor - Ende der 50er Jahre - gingen in Heidelberg und Köln zwei unterschiedliche Notarztdienst-Modelle in die Erprobungsphase. Während in Heidelberg ein „Klinomobil“ mit einer siebenköpfigen OP-Besatzung an die Unfallstelle ausrückte, wurde in Köln das „stationäre System“ initiiert, welches auch heute noch - vornehmlich in Großstädten etabliert ist. Dabei rückte im Alarmierungsfall ein an einer Klinik stationiertes 
Rettungsfahrzeug besetzt mit zwei Sanitätern und einem Arzt zum Notfallgeschehen aus [14].

Fast zeitgleich setzten im Osten Deutschlands die zwei jungen Magdeburger Ärzte, der Anästhesist Röse und der Chirurg Lembcke, in Zusammenarbeit mit der Berufsfeuerwehr das Konzept eines arztbesetzten Rettungsmittels um [10].

Unter Berücksichtigung der Erfahrungen mit dem zuvor erwähnten Klinomobil und dem Kölner stationären System entwickelte der Heidelberger Chirurg Eberhard Gögler im Jahre 1964 eine weitere, bis heute prägende Variante des Notarzt-Systems mit einem schnellen und wendigen Einsatzfahrzeug für den Klinikarzt. Dies gilt als die Geburtsstunde des "NotfallArztwagens". Es stellte das noch heute in seiner ursprünglichen Form verwendete Notarzteinsatzfahrzeug (NEF) dar. Parallel zu diesem Fahrzeug wurde synchron der Unfallrettungswagen (URW) an die Einsatzstelle entsandt. Beide Fahrzeuge wurden 1959 mit festen Besatzungen in den Rettungsdienst integriert. Dadurch war das Einsatzkonzept des "Rendezvous-Systems" geschaffen. Durch die getrennte Anfahrt von Notarzt und Rettungsdienst, konnte so eine zielgerichtete und flexiblere Disposition der Ärzte stattfinden. Der Fokus lag nunmehr auf der Versorgung des Patienten am Notfallort (sog. „stay and play“-System). Diese Strategie beinhaltete - nach Herstellung der Transportfähigkeit - die Verbringung des Patienten in die nächstgelegene geeignete Klinik unter kontinuierlicher Überwachung der Vitalparameter.

Ab 1967 wurde das deutsche Rettungswesen durch Ahnefeld und Frey verstärkt ausgebaut [1]. Sie prägten den Begriff der „Rettungskette“ und machten sich zum Ziel, die Bevölkerung, welcher eine nicht unerhebliche Aufgabe am Notfallort zukommt, auf breiter Basis in „Erster Hilfe" auszubilden [29].

Parallel zu den Entwicklungen in der Bundesrepublik Deutschland wurde in der ehemaligen Deutschen Demokratischen Republik (DDR) das System der Schnellen Medizinischen Hilfe (SMH) als Kooperation des Deutschen Roten Kreuzes und dem Ministerium für Gesundheit im Sinne einer zentral organisierten staatliche Aufgabe etabliert.

In Leipzig begannen im März 1964 die Anästhesisten Splith und Heidel mit einem Pilotprojekt unter dem Namen „Dringliche Medizinische Hilfe“ (DMH). Hierbei handelte es sich um einen umgebauten Krankentransportwagen (KTW) der - auch aus heutiger Sicht - eine 
allumfassende medizinische Ausstattung aufwies. Diese Fahrzeuge - zunächst zwei Fahrzeuge für das Stadtgebiet mit damals 560.000 Einwohnern - waren mit jeweils zwei Krankentransporteuren und einem Arzt besetzt. Krankentransporteure waren Mitarbeiter des Deutschen Roten Kreuzes der DDR mit einer beliebigen Berufsausbildung und einer geringgradigen Sanitätsausbildung von 160 Stunden. Neben dem Leipziger DMH Modell gab es ähnliche Initiativen in Städten wie Greifswald, Jena, Dresden oder Chemnitz. Aus diesen Erfahrungen heraus entstand im Jahre 1967 eine erste staatliche Direktive, die Empfehlungen zur Bildung einzelner Modelle für Großstädte und Ballungsgebiete vermittelten. Weitere 10 Jahre später wurde die arztbesetzte Dringliche Medizinische Hilfe in das System der Schnellen Medizinischen Hilfe (SMH) integriert. Weitere Komponenten wie die Leitstellen, die einheitliche Notrufnummer 115, der Krankentransport, der Dringliche Hausbesuchsdienst (DHD) und die Rettungsstellen trugen zur Vervollständigung einer flächendeckenden Notfallversorgung bei [10].

In diesem Zusammenhang besonders hervorzuheben ist die gemeinsame Disposition von Notfallversorgung durch die SMH und Dringlichkeitsversorgung durch den DHD, welche von der zuständigen Leitstelle übernommen wurde.

Damit musste nicht der Patient bzw. der Anrufer selbst den Notfall klassifizieren, sondern das geschulte Leitstellenpersonal entschied anhand der übermittelten Daten welche Versorgungsstufe initiiert wurde.

Die Ära der Luftrettung in Deutschland begann im Jahr 1957 mit dem "Such- und Rettungsdienst" der Bundeswehr. 1970 wurde schließlich der erste zivile Rettungshubschrauber offiziell seiner Bestimmung übergeben. Seit dieser Zeit ist die Luftrettung ein fester Bestandteil des Rettungsdienstes [5]. 


\subsection{Aktuelles Rettungsdienstsystem}

Setzt man über die bundesweite Telefonnummer 112 einen Notruf ab, wird man unmittelbar mit einem Mitarbeiter der jeweiligen Rettungsleitstelle verbunden. Dieser nimmt nach standardisierter Abfrage der verschiedenen Notfallparameter die häufig parallele Disposition eines Rettungsmittels (meist RTW - Rettungswagen) und ggf. eines zusätzlichen Notarztes (NEF - Notfalleinsatzfahrzeug/Rettungshubschrauber) vor. In einigen Regionen wird auch ein Notarztwagen (NAW), ein arztbesetzter Rettungswagen, entsandt. In manche Leitstellen ist auch die Disposition des kassenärztlichen Bereitschaftsdienstes integriert, sodass - nach entsprechender telefonischer Triage - auch die Entsendung eines Dienstarztes mit entsprechend der medizinischen Dringlichkeit längerer Hilfsfrist möglich ist.

Stellt das entsendete Rettungsteam vor Ort die Indikation zur stationären Aufnahme bzw. einer unmittelbaren weiteren ärztlichen Behandlungsnotwendigkeit folgt der Transport des Patienten mit oder ohne Notarztbegleitung in das nächstgelegene geeignete Krankenhaus.

Ein vergleichbar flächendeckendes System mit permanenter notärztlicher Verfügbarkeit wie in Deutschland existiert nur in Österreich, der Schweiz, Frankreich, Luxemburg, Dänemark und Finnland. Alle anderen europäischen Länder bzw. westliche Industrienationen verfügen über verschiedene Ambulanzsysteme mit der Handlungsprämisse des schnellen Patiententransportes, mit speziell geschultem nicht-ärztlichem Rettungsdienstfachpersonal (Paramedics). Nur vereinzelt existieren in Ballungszentren oder per Hubschrauber arztgestützte Systeme.

In der Stadt Leipzig wird der Rettungsdienst auf der Grundlage vertraglicher Vereinbarungen zwischen der Stadt und den Leistungserbringern (Feuerwehr, Arbeiter-Samariter-Bund, Deutsches Rotes Kreuz, Johanniter Unfallhilfe, Ambulanz Service Gesellschaft Leipzig GmbH, Krankentransport Ost-West $\mathrm{GmbH}$ ) durchgeführt. Die Organisation obliegt der Branddirektion Leipzig, die Alarmierung und Disposition erfolgt über die Feuerwehr- und Rettungsleitstelle. Jedem der o.g. Anbieter ist ein Rettungsdienstbezirk zugeordnet, in welchem insgesamt 18 Rettungswagen (RTW) und 7 Notarzteinsatzfahrzeuge im Rendezvous-Verfahren zum Einsatz kommen. 


\subsection{Ableitung der Studie / Wissenschaftlicher Hintergrund}

Der Rettungsdienst nimmt eine wichtige Funktion im deutschen Gesundheitswesen ein.

Jeder Bürger hat einen gesetzlich garantierten Anspruch auf eine flächendeckende, Hilfsfristorientierte, qualifizierte Hilfe, die dem jeweiligen Stand des medizinischen Wissens und der Technik entspricht und rund um die Uhr an jedem denkbaren Ort sicherzustellen ist [30]. Vor dem Hintergrund dieser Herausforderungen an unser Notfallrettungssystem stellt sich die Frage wie lange und in welcher Form dies in der Bundesrepublik im Hinblick auf eine qualifizierte und fristgerechte Rettung für jeden Bürger zu jeder Zeit umsetzbar ist. Ein funktionstüchtiger, nachhaltig tragfähiger und effizienter Rettungsdienst liegt selbsterklärend im allgemeinen Interesse.

Im deutschen Rettungsdienst wurde jedoch seit Jahren über eine kontinuierlich steigende Zahl von Notarzteinsätzen berichtet [3]. Insbesondere im Leipziger Stadtgebiet kam es in den Jahren nach der deutschen Wiedervereinigung $1990 \mathrm{zu}$ einem drastischen Anstieg der Notarzteinsatzzahlen, welche zudem weit über dem bundesdeutschen Durchschnitt lagen und auch weiterhin liegen [10]. Betrachtet man die Prognosen des Zensus und die Angaben des Statistischen Bundesamts genauer, so wird sich bei einer allgemeinen Reduktion der Gesamtbevölkerung die Geburtenrate bis 2050 auf etwa 0,5 Mio./Jahr reduzieren und es zu diesem Zeitpunkt etwa doppelt so viele 60-Jährige im Vergleich zu Neugeborenen geben [42]. Jener demografische Wandel ist zwar ein gesamtgesellschaftliches und bundesweites Phänomen, das jedoch durch regionale Unterschiede - verursacht durch sozial- und arbeitsmarktpolitisch bedingte Abwanderung und Umsiedlung - gekennzeichnet ist. So ist insbesondere in den östlichen Bundesländern ein deutlicher Anstieg des Anteils der älteren Bevölkerung zu verzeichnen (Gesamtanteil Bevölkerung >67 Jahre: Flächenländer West $29,0 \%$ vs. $34,5 \%$ Flächenländer Ost [44]) und betrifft somit auch das Stadtgebiet von Leipzig.

Neben steigenden Einsatzzahlen sind auch deutliche Veränderungen hinsichtlich des Einsatzspektrums notärztlicher Einsätze zu beobachten. Dominierten in früheren Zeiten traumatologische Notfälle, stehen heutzutage insbesondere internistisch-neurologische Krankheitsbilder, häufig mit entsprechend chronischen Verlaufsformen, im Mittelpunkt der 
notärztlichen Tätigkeit. Dies hat zur Folge, dass die Notfallmedizin immer mehr dort nachgefragt wird, wo Lücken im Versorgungsnetz bestehen [31].

Trotz dieser vielerorts beobachteten und diskutierten Entwicklung steigender Einsatzzahlen und geänderter Einsatzspektren gab es bisher nur wenige Daten aus großen Patientenkollektiven, die diese Veränderungen über einen größeren Zeitraum darstellten und entsprechende Rückschlüsse auf deren Ursachen und Lösungsmöglichkeiten zur Reduktion zogen. Eine Reduktion bzw. eine Stagnation der Einsatzzahlen und das Hinterfragen der tatsächlichen notärztlichen Erfordernis der geleisteten und zu leistenden Einsätze ist aber vor dem Hintergrund der ökonomischen Effizienzsteigerung und den limitierten, sich häufig an der Belastungsgrenze befindlichen klinischen Notfallversorgungsstrukturen, erstrebenswert [28].

\section{Ziele der Studie}

Im Rahmen der von uns durchgeführten, retrospektiven Studie sollten die Notarzteinsätze im Stadtgebiet Leipzig im Zeitraum 2003-2013 analysiert und hieraus ggf. Gründe kontinuierlich steigender Einsatzzahlen identifiziert werden. Es wurde jeweils das 1. Quartal beider Vergleichsjahre ausgewertet. Von besonderem Interesse war hierbei die Entwicklung des Patientenkollektivs hinsichtlich Alter, Geschlecht, Erkrankungsschwere und dem Anteil der im Pflegeheim betreuten Patienten verbunden mit der Frage nach einer möglichen Änderung des Diagnosespektrums. Es sollte der Umfang der notärztlichen Versorgung in Bezug auf die Invasivität der Maßnahmen oder dem Medikamentengebrauch untersucht werden. Zudem sollten eventuelle Änderungen hinsichtlich des Einsatzortes und mögliche Tendenzen bezüglich der Alarmierungs- und Einsatzzeiten heraus kristallisiert werden. Anhand der Ergebnisse sollten Rückschlüsse auf mögliche Umstrukturierungsmaßnahmen zur optimalen Nutzung der vorhandenen Ressourcen gezogen werden, sich Anhaltspunkte für die zukünftige materielle und personelle Ausstattung des Rettungsdienstes und die Qualifikation des beteiligten ärztlichen und nicht-ärztlichen Personals ergeben und Schlussfolgerungen zur Optimierung der Zusammenarbeit an der Schnittstelle Notarztdienst und Zentrale Notaufnahme gezogen werden.

Der in der Arbeit gewählte Beobachtungszeitraum von 10 Jahren gründet im Wesentlichen auf zwei Faktoren. Zum einen beschreibt eine vorherige Analyse die Einsatzentwicklung bis 
Anfang der 2000er Jahre [3], so dass vor dem Hintergrund sich ändernder Rahmenbedingungen (z.B. Einführung der DRG-Fallpauschalen) eine Beobachtung der nachfolgenden 10 Jahre aus Sicht der Autoren von besonderem Interesse erschien. Zum anderen stellt das Beobachtungsintervall bei Initiierung der Analyse den zur Datenakquise (2013) größtmöglichen retrospektiven Zeitraum dar, da die Protokolle nach jeweils 10 Jahren nicht mehr archivierungspflichtig sind und entsprechend entsorgt wurden.

Die zur Verfügung stehenden Daten beliefen sich jeweils auf das erste Quartal der Jahre 2003 und 2013. Ausgewertet wurden alle Notarztprotokolle des bodengebundenen Rettungsdienstes. Im Rahmen der statistischen Beratung durch das IMISE (Institut für medizinische Informatik, Statistik und Epidemiologie der Universität Leipzig) wurde der Vergleich des jeweils ersten Quartals bei einer Datenmenge von jeweils mehr als 6000 Protokollen bestätigt. Ein wesentlicher Signifikanzgewinn bei einer Erhöhung der Datenmenge wurde nicht in Aussicht gestellt. Zur eigenen Überprüfung fand zudem eine Berechnung der aus den Quartalsdaten hochgerechneten Jahreseinsatzzahlen statt, welche dann mit den real erhobenen Jahreseinsatzzahlen verglichen wurden. Hierbei zeigte sich nur eine geringe Abweichung zwischen berechneten und realen Daten, so dass die Schlussfolgerung von den Quartalsdaten auf die Jahresdaten reliabel erscheint und eine Verzerrung durch jahreszeitabhängige Erkrankungsspezifika nicht relevant zu sein scheint.

Im Untersuchungszeitraum gab es keine grundsätzlichen strukturellen Anpassungen, insbesondere keine standardisierte Notrufabfrage und keine Änderungen im Notarzteinsatzkatalog, sodass die Vergleichbarkeit beider Zeiträume gegeben scheint. Lediglich die Anzahl der Rettungsmittel änderte sich in Anpassung an die gestiegenen Einsatzzahlen. Des Weiteren erfolgten seitens der Leitstelle Änderungen der Alarmierungsstichworte im Hinblick auf die optimierte Zuordnung zu bestimmten Krankheitsbildern. Dies blieb ohne Einfluss auf die Gesamtzahl der Einsätze und die Auswertung im Sinne der Studie. 


\subsection{Publikation der Studie}

Die Veröffentlichung der vorliegenden Studie erfolgte in der nach dem peer-reviewVerfahren arbeitenden und international gelisteten deutschsprachigen Fachzeitschrift DER ANAESTHESIST (Springer Medizin, Springer Verlag GmbH, Heidelberg). Für die Auswahl des Publikationsorgans war entscheidend, dass sich die vorliegende Untersuchung mit Spezifika des deutschen Rettungswesens befasst und daher aufgrund der international z.T. gravierenden Systemunterschiede in der präklinischen Notfallmedizin vorrangig für den deutschsprachigen Raum von Interesse ist.

Die vorliegende Publikation wurde von Katja Bader entworfen und bis zur endgültigen Form weiterbearbeitet. Alle beteiligten Autoren haben den Entstehungsprozess vom Entwurf des Studiendesigns, bis zur Datenerhebung kritisch begleitet und das Manuskript ergänzt und korrigiert. Eine Abdruckgenehmigung des Verlages zum Nachdruck des Zeitungsbeitrages im Rahmen der Dissertation liegt vor.

Vor der Veröffentlichung der Originalarbeit wurden Ergebnisse von Zwischen- und Endauswertungen auf der Jahrestagung der Deutschen Gesellschaft für Internistische Intensivmedizin und Notfallmedizin präsentiert und als Abstract veröffentlicht. Weitere Angaben hierzu sind im Anhang zu finden. 


\title{
2. Publikation
}

\section{Entwicklung bodengebundener Notarzteinsätze im Stadtgebiet Leipzig von 2003 bis 2013}

\author{
K. Bader ${ }^{1} \cdot$ M. Bernhard ${ }^{1} \cdot$ A. Gries ${ }^{1} \cdot$ M. Kaul ${ }^{2} \cdot$ R. Schröder $^{2} \cdot$ A. Ramshorn-Zimmer ${ }^{1}$ \\ ${ }^{1}$ Zentrale Notaufnahme, Universitätsklinikum Leipzig, Leipzig, Deutschland \\ ${ }^{2}$ Branddirektion, Stadt Leipzig, Leipzig, Deutschland
}

erschienen in der Fachzeitschrift DER ANAESTHESIST

Springer Medizin, c/o Springer Verlag $\mathrm{GmbH}$, Heidelberg

(Abdruck mit freundlicher Genehmigung des Verlages)

DOI:10.1007/s00101-017-0393-2

PubMed ID: 29230501 


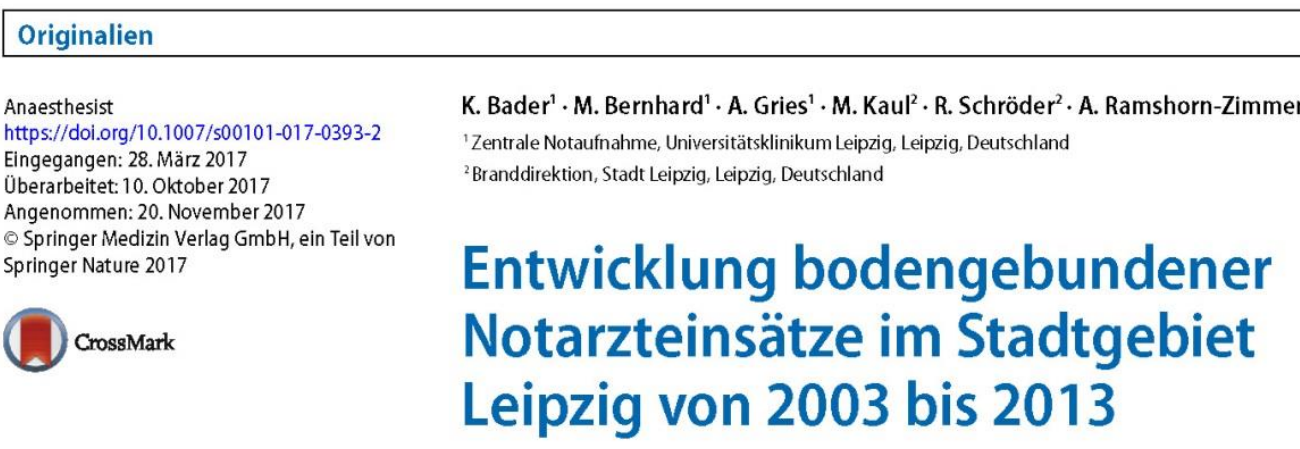

\section{Einleitung}

Im bundesdeutschen Notarztdienst werden kontinuierlich steigende Einsatzzahlen berichtet [2]. So stieg gemäß den Zahlen der Bundesanstalt für Straßenwesen von $2004 / 2005$ bis 2013 die ge samtdeutsche Notarzteinsatzrate von 28 auf 34 (Steigerung um $21 \%$ ) [6]. Als Ursache der steigenden Einsatzzahlen werden die Reduktion alternativer, auch ambulanter Versorgungsstrukturen und die Veränderung der prähospitalen Notfall versorgung diskutiert [26]. Als weitere Ursachen für ein höheres Patientenaufkommen im Rettungs-und Notarztdienst wurden bereits der demografische Wandel mit einer Zunahme multimorbider Patienten in der Gesamtbevölkerung, die Qualität der medizinischen Versorgung in Pflegeheimen und der zunehmende Medieneinfluss im Hinblick auf Früh symptome verschiedener Krankheitsbilder angeführt $[1,18,20]$. Vor diesem Hintergrund ist auch zukünftig mit einem weiteren Zuwachs an Notarzteinsät zen zu rechnen $[23,32]$.

Betrachtet man die Prognosen des Zensus und die Angaben des Statistischen Bundesamts genauer, so wird sich bei einer allgemeinen Reduktion der $\mathrm{Ge}$ samtbevölkerung die Geburtenrate bis 2050 auf etwa 0,5 Mio./Jahr reduzieren und es zu diesem Zeitpunkt etwa doppelt

Teile der vorliegenden Arbeit entstanden im Rahmen der Dissertation von Frau Katja Bade an der Medizinischen Fakultät der Universität Leipzig und wurden während der Jahrestagung der Deutschen Gesellschaft für Internistische der Deutschen Gesellschaft fur Internistische
Intensiv- und Notfallmedizin am 19.06.2015 präsentiert.

Published onlinc: 11 December 2017 so viele 60-Jährige im Vergleich zu Neugeborenen geben [31]. Der Notarzt wird sich also mit einer kontinuierlichen Steigerung an älteren Notfallpatienten mit hoher Erkrankungsschwere konfrontiert sehen [22].

In den Jahren nach der deutschen Wiedervereinigung 1990 sind die Notarzteinsatzzahlen und damit auch die Bindung entsprechender für die Notfallversorgung erforderlicher struktureller und personeller Ressourcen im Leipziger Stadtgebiet drastisch angestiegen [7] Vor dem Hintergrund der Diskussion um eine Steigerung der ökonomischen Effizienz und auch im Hinblick au die sich häufig an der Belastungsgrenze befindlichen klinischen Notfallversorgungsstrukturen müssen die stetig steigenden Einsatzzahlen und auch die tatsächlichen Erfordernisse der notärztlichen Versorgung analysiert werden um eine rationale Bewertung vornehmen und Lösungsansätze entwickeln zu können [21]. Trotz einer vielerorts beobachteten kontinuierlichen Steigerung der Notarzteinsatzzahlen liegen hierzu in Deutschland nur wenige Daten aus großen Patientenkollektiven vor, die diese Veränderungen über einen größeren Zeitraum darstellen.

In der vorliegenden Untersuchung sollen daher patientenbezogene sowie einsatztechnische Daten und die durchgeführten notärztlichen Maßnahmen im bodengebundenen städtischen Notarztsystem in Leipzig retrospektiv über einen Zeitraum von 10 Jahren analysiert werden.

\section{Material und Methode \\ Untersuchungsgebiet und notärztliche Infrastruktur}

Die Stadt Leipzig ist die größte Stadt im Freistaat Sachsen und die zehntgrößte Stadt der Bundesrepublik Deutschland. Sie erstreckt sich über ein Gebiet von $297,8 \mathrm{~km}^{2}$. Im Jahr 2003 lebten 497.531 Einwohner in der als Handelsund Wissenschaftsmetropole bekannten Stadt, 2013 waren es 531.562 bei vergleichbarer Stadtfläche (29.761 vs. 29.739 ha). Der Rettungsdienst wird auf der Grundlage vertraglicher Vereinbarungen zwischen der Stadt Leipzig und den Leistungserbringern (Feuerwehr, Arbeiter-Samariter-Bund, Deutsches Rotes Kreuz, Johanniter Unfallhilfe, Ambulanz Service Gesellschaft Leipzig GmbH, Krankentransport Ost-West $\mathrm{GmbH}$ ) durchgeführt. Die Organisation obliegt der Branddirektion Leipzig; Alarmierung und Disposition erfolgen über die Feuerwehr- und Rettungsleitstelle. Jedem der oben genannten Anbieter ist ein Rettungsdienstbezirk zugeordnet, in dem insgesamt 18 Rettungswagen (RTW) und 7 Notarzteinsatzfahrzeuge im Rendezvous-Verfahren zum Einsatz kommen.

Im Jahr 2003 wurden 5 Notarzteinsatzfahrzeuge rund um die Uhr und 2013 sieben Notarzteinsatzfahrzeuge im Tagdienst (07:00-18:59 Uhr) und 5 Notarzteinsatzfahrzeuge im Nachtdienst (19:00-06:59 Uhr) betrieben. Einsätze der in Leipzig stationierten Luftrettungsmittel (ADAC Luftrettung gGmbH, Christoph 61 und 63, Luftrettungsstati- 
Originalien

on Leipzig/Halle) wurden im Sinne der Fragestellung nicht berücksichtigt. Fehlfahrten bzw. Einsatzabbrüche wurden in der Analyse ausgeschlossen. Wesentliche strukturelle Änderungen im Sinne einer Änderung des Notarztindikationskataloges oder einer strukturierten/ standardisierten Notrufabfrage fanden im Beobachtungszeitraum nicht statt. Es wurden lediglich die Alarmierungsstichworte modifiziert und die Zahl der Rettungsmittel, wie zuvor beschrieben, erhöht.

\section{Datenerfassung und -analyse}

In einer retrospektiven Untersuchung wurden mit Zustimmung der Ethikkommission der Medizinischen Fakultät der Universität Leipzig (Az. 274-1307102013) die Protokolle der bodengebundenen Notarzteinsätze im Stadtgebiet Leipzig jeweils des Zeitraums 01. Januar bis 31. März (I. Quartal) der Jahre 2003 und 2013 erfasst und verglichen.

Der Datenauswertung lagen die Notarzteinsatzprotokolle (2003: DIVIDOK ${ }^{\otimes}$ Version EPRO 2.5; 2013: DIVIDOK Version EPRO 4.2, Fa. DokuFORM Verlags-GmbH, Lübeck) der einzelnen, bodengebundenen Notarztstandorte im Stadtgebiet Leipzig zugrunde. An einem der Notarztstandorte wurden 2013 die Einsatzprotokolle elektronisch erfasst und als PDF gespeichert (MedicalPad, Version 7 Leipzig, Fa. TechToGo, Hamburg), sodass im Rahmen der Untersuchung auf diese Dokumente zurückgegriffen werden konnte. Folgende Daten wurden den Notarzteinsatzprotokollen der Jahrgänge 2003 und 2013 entnommen und weiteranalysiert: patientenbezogene Daten (Alter, Geschlecht), Einsatzstichwort, -zeitpunkt, -ort, Notarztqualifikation, Transportziel, Notarztdiagnose, notärztliche Interventionen, Erkrankungs- und Verletzungsschwere gemäß National Advisory Committee for Aeronautics (NACA) Score und Bewusstseinslage gemäß Glasgow Coma Scale (GCS). Die Notarzteinsatzrate ([Notarzteinsätze/Jahr und Einwohnerzahl] $\cdot 1000)$ beider Zeiträume wurde berechnet [5].

Der Einsatzzeitpunkt wurde den Zeiträumen 07:00-18:59 Uhr(Tagdienst) oder 19:00-06:59 Uhr (Nachtdienst) zugeordnet. Als Einsatzort wurden erfasst: Patientenwohnung, Pflegeeinrichtung, öffentlicher Raum und Institutionen (Klinik, Arztpraxis). Als Notarztqualifikation wurden der Weiterbildungsstand (Weiterbildungsassistent vs. Facharzt) und die jeweilige Fachrichtung (z.B. Anästhesie, innere Medizin, Chirurgie) erfasst. Als Transportziel wurde bei Krankenhauszuweisungen zwischen Maximal- und Regelversorger differenziert bzw. eine ambulante Weiterversorgung des Patienten dokumentiert.

Zur Bestimmung der Erkrankungsbzw. Verletzungsschwere (vitale Gefähr dung) wurde der durch den Notarzt dokumentierte NACA-Score verwendet. Bei fehlender Dokumentation wurde dieser bei vorliegenden objektiven $\mathrm{Pa}$ rametern wie Transportziel, Vitalparametern und der Einsatzbeschreibung retrospektiv ermittelt.

Ergänzend wurde aus den notärztlich dokumentierten Daten (GCS [Summenscore], Herzfrequenz [x/min], Atemfrequenz [x/min], Herzrhythmus, Schmerz, Blutdruck $[\mathrm{mm} \mathrm{Hg}]$, pulsoxymetrische Sauerstoffsättigung [\%]) der Mainzer Emergency Evaluation Score (MEES, [17]) kalkuliert. Eine vitale Bedrohung wurde bei einem Punktwert von 1 („MEES*“) bei einem der aufgeführten Parameter angenommen; die übliche Angabe eines $\triangle \mathrm{MEES}$ war aufgrund fehlender Übergabe- bzw. innerklinischer Verlaufsparameter nicht möglich.

Um eine Zuordnung $\mathrm{zu}$ verschiedenen Einsatzkategorien vornehmen zu können, wurden die durch den Notarzt dokumentierten Erstdiagnosen des DIVI-Protokolls wie folgt subsumiert: internistischer Notfall (Angina pectoris, Herzinfarkt, Rhythmusstörung, Lungenembolie, Lungenödem, Herzinsuffizienz, hypertensive Krise, Orthostase, Herz-Kreislauf-Stillstand, Pacemaker/Implantierter Karsioverter/ Defibrillator(PM/ICD)-Dysfunktion, sonstige Erkrankungen Herz/Kreislauf, Asthma, exazerbierte Chronisch obstruktive Lungenerkrankung (COPD), Aspiration, Pneumonie/Bronchitis, Hyperventilationstetanie, Pseudokrupp/ Epiglottitis, sonstige Erkrankungen der Atmung, Blutzuckerentgleisung, Ex- sikkose, sonstige Erkrankungen des Stoffwechsels, akutes Abdomen, gastro intestinale Blutung, abdominelle Kolik und sonstige Erkrankungen des Abdomens, anaphylaktische Reaktion, Un terkühlung, Ertrinken, sonstige Intoxikationen, Tumorleiden/Finalstadium Rauchgasinhalation), traumatologischer Notfall (Verletzungen jeglicher Art [of fen/geschlossen, leicht-schwer], Verbrennungen, Elektrounfall und Polytrauma), pädiatrisch/geburtshilflicher Notfall (Evaluation nach Alter und Diagnose Geburt, Sudden Infant Death Syndrom [SIDS]), neurologischer Notfall (Insult, Transitorische Ischämische Attacke [TIA], Blutung, Krampfanfall, sonstige Erkrankungen des Zentralen Nervensystems [ZNS]), psychiatrischer Notfall (Psychose, Depression, Manie Intoxikation, Entzug, Suizidversuch, sonstige Erkrankungen Psychiatrie) und sonstiger Notfall (vaginale Blutung und sonstige gynäkologische Erkrankungen, unbekannte Erkrankung, Freitexteinga be). Die den Notarzteinsatzprotokollen der Jahre 2003 und 2013 entnommenen anonymisierten Daten wurden in elek tronischer Form (Microsoft ${ }^{\circledR}$ Excel 2010 Deutschland) erfasst und im Anschlus mithilfe von SPSS (IBM-Statistics, Ver sion 22) weiter statistisch ausgewertet.

\section{Statistik}

Nach Untersuchung auf Normalvertei lung mithilfe des Kolmogorov-SmirnovTests wurden die Daten mithilfe des $\chi^{2}$ Tests, des Student-t-Tests, des WilcoxonTests und des Exakten Tests nach Fischer untersucht. Alle statistischen Analysen wurden unter Anwendung der StatistikSoftware IBM SPSS-Statistics durchgeführt. Die Angabe der Daten erfolgte als absolute Zahlen (Median, Mittelwert $[\mathrm{MW}] \pm$ Standardabweichung [SD], Mi nimal- [min] bis Maximalwert [max]) bzw. als Prozent. Eine Fehlerwahrscheinlichkeit $p<0,05$ wurde als statistisch signifikant gewertet. 
Zusammenfassung · Abstract

Anaesthesist https://doi.org/10.1007/s00101-017-0393-2

๑) Springer Medizin Verlag GmbH, ein Teil von Springer Nature 2017

K. Bader · M. Bernhard · A. Gries · M. Kaul · R. Schröder · A. Ramshorn-Zimmer

Entwicklung bodengebundener Notarzteinsätze im Stadtgebiet Leipzig von 2003 bis 2013

Zusammenfassung

Hintergrund. Im deutschen Rettungsdienst wird über die kontinuierlich steigende Zah von Notarzteinsätzen berichtet. Bisher lagen jedoch kaum Daten aus Großstädten vor, die diese Veränderungen über einen längeren Zeitraum darstellen.

Material und Methode. In einer retrospektiven Untersuchung wurden die Notarzteinsätze jeweils des Zeitraums 01 . Januar bis 31. März der Jahre 2003 und 2013 der Stadt Leipzig ausgewertet. Neben der Notarzteinsatzrate (Notarzteinsätze/1000 Einwohner und Jahr) wurden die Erkrankungs- und Verletzungsschwere (National Advisory Committee of Aeronautics[NACA]-Score), der Einsatzort und der Versorgungsgrad der Zielklinkerfasst.
Ergebnisse. Die Zahl der Notarztein sätze in den Beobachtungszeiträumen nahm zwischen 2003 und 2013 um $24 \%$ ( $n=6030$ vs. $n=$ 7470) und die Notarzteinsatzrate um $21 \%$ (48 vs. 58) zu. Bei steigendem Patientenalter ( 66 vs. 70 Jahre) stieg der Anteil hochbetagter (Alter $\geq 85$ Jahre: $11 \%$ vs. $16 \%(p<0,01)$ ) und der Anteil vital gefährdeter Patienten (NACA IV-VI: $14 \%$ vs. $16 \%(p<0,01))$. Notarzteinsätze in Pflegeheimen vervierfachten $\operatorname{sich}(n=175(3 \%)$ vs. $n=750(10 \%), p<$ (1) 1 il); $0,01)$; die Zahl der Krankenhauseinweisunge stieg an $(n=3049(51 \%)$ vs. $n=4738(66 \%)$,
$p<0,01)$. Hierbei nahm maßgeblich der Anteil Maximalversorgern zugeführter Patienten zu $(n=1742(29 \%)$ vs. $n=3436(46 \%), p<0,01)$.
Schlussfolgerung. Im Stadtgebiet Leipzig wurden zwischen 2003 und 2013 steigende Notarzteinsatzzahlen und ein Anstieg schwer erkrankter und geriatrischer Patienten nachgewiesen. Die im Vergleich zum Bundesdurchschnitt nahezu doppelt so hohe Bundesdurchschnitt nahezu doppelt so hoh
Notarzteinsatzrate kann jedoch auf Defizite im Bereich der ambulanten Grundversorgung und Optimierungsmöglichkeiten bei der Disposition der Rettungsleitstelle hinweisen.

Schlüsselwörter

Patientenspektrum - Notarztdienst Notärztliche Qualifikation - Geriatrische Notfallpatienten - Epidemiologie

\section{Development of ground-based physician-staffed emergency missions in the city of Leipzig from 2003} to 2013

Abstract

Background. The annual number of physician-based emergency missions reported is continuously increasing. Data from large cities concerning this development over long periods is sparse.

Material and methods. In this retrospective study the charts of all ground-based physicianstaffed emergency missions in the city of Leipzig for the first quarters of 2003 and 2013 were analyzed. Patient characteristics, injury and illness severities, mission location, hospital admission rate, as well as emergency interventions were collated. The emergency mission rate was calculated as rescue missions per 1000 inhabitants per year.

Results. The number of physician-staffed emergency missions increased by approximately $24 \%$ between 2003 and $2013(6030$ vs. 7470 , respectively). The emergency mission rate was 48 vs. 58 in the 2 study periods. The median patient age increased from 66 to 70 years. The number of geriatric patients (age $\geq 85$ years: $n=650(11 \%)$ vs. $n=1161(16 \%), p<0.01)$ a so increased. The corresponding number of emergency missions in nursing homes showed a fourfold $(n=175,3 \%$ vs. $n=750,10 \%, p<0.01)$. The percentage of hospital admissions also increased $(n=3049,51 \%$ vs. $n=4738,66 \%$, $p<0.01$ ) A change in patient distribution to $p<0.01$. Ach level Ihospitals was noticed ( $n=1742,29 \%$ vs. $n=3436,46 \%, p<0.01$.

Conclusion. The findings suggest that the necessity for the high number of physicianstaffed emergency missions should be verified, especially in the context of strained emergency healthcare resources. The basis of an optimized use of resources could be a better inclusion of alternative, especially ambulant, healthcare structures and the implementation of a structured emergency call questionnaire accompanied by a more efficient disposition of the operating resources, not least in view of the economic aspects. Taking the concentrated patient allocation to level 1 hospitals into consideration, there is a need for optimized patient distribution strategles to minimize the overload of individual institutions and thereby improve the general quality of care at the interface between preclinical and clinical emergency medicine.

\section{Keywords}

Spectrum of patients - Physician-staffed emergency medical services - Emergency physician qualification - Geriatric patients. Epidemiology

\section{Ergebnisse}

\section{Einsatzzahlen}

Die Anzahl der Notarzteinsätze im Stadt gebiet Leipzig nahm von 6030 Einsät zen im I. Quartal 2003 auf 7470 Einsätze im I. Quartal 2013 signifikant um $24 \%$ zu. Damit wurden 2003 im Durch schnitt 65 Einsätze/Tag und 201384 Ein sätze/Tag absolviert. Die Notarzteinsatz- rate stieg im beobachteten Zeitraum von 48 Einsätzen/1000 Einwohner und Jahr auf 58 Einsätze/1000 Einwohnerund Jahr $(p<0,001)$. Die Nachalarmierungsquote durch Kliniken oder niedergelassene Ärzte sank im Beobachtungszeitraum (2003: $3,2 \%$ vs. $2013: 1,6 \%, p<0,001$ ) Die Nachforderungshäufigkeit eines Notarztes durch den Rettungsdienst nahm hingegen leicht zu (2003: $10 \%$ vs. 2013 $11,7 \%, p<0,05)$. Hinsichtlich der Ta- geszeit der Einsätze ergaben sich zwischen 2003 und 2013 keine signifikanten Unterschiede, und der Anteil betrug im Tagdienst $59,2 \%$ bzw. 58,4\%.

\section{Patientencharakteristika}

Angaben zum Alter lagen 2003 bei 6005 Patienten $(99,6 \%)$ und 2013 bei $7442 \mathrm{~Pa}$ tienten $(99,6 \%)$ vor. Beim Alter der versorgten Patienten zeigten sich signifikan- 


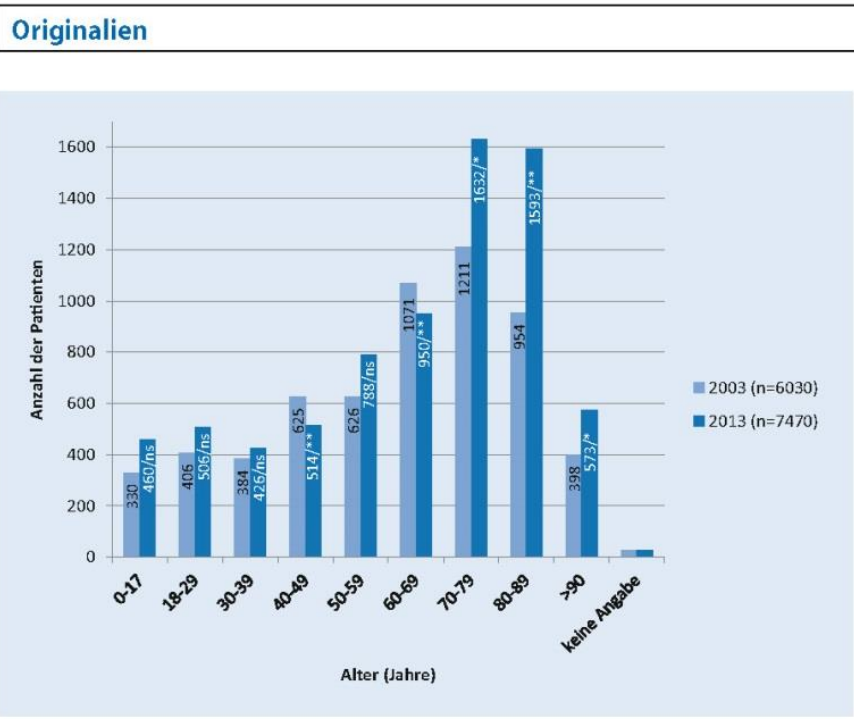

Abb. $1 \Delta$ Vergleich der Altersstruktur der notärztlich versorgten Patienten des I. Quartals 2003 und $2013\left({ }^{*} p<0,05 ;{ }^{* *} p<0,001 ; n\right.$ s nicht signifikant)

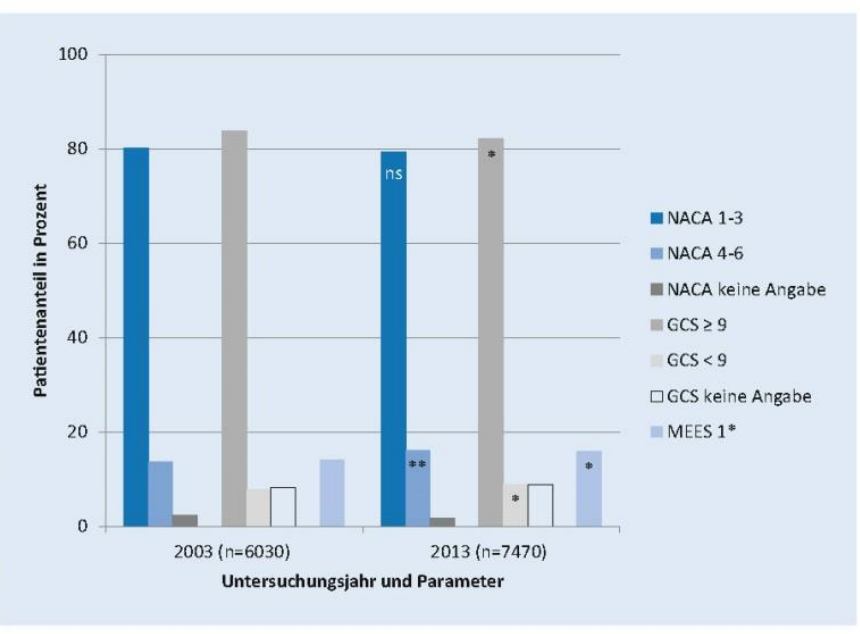

Abb. 2 A Prozentualer Vergleich der Erkrankungs-undVerletzungsschwere der notärztlich versorgten Patienten gemäß NACA-Score I-III/IV-VI und fehlender Angaben, GCS $\geq 9 / G C S<9$ und fehlender Angaben, Mainzer Emergency Score (MEES) mit angezeigter Vitalbedrohung (MEES $1^{*}$ ) des I. Quartals 2003 vs. $2013\left({ }^{*} p<0,05 ;{ }^{* *} p<0,001 ; n\right.$ s nicht signifikant), NACA $=$ National Advisory Comittee of Aeronautics Score, GCS = Glasgow Coma Skala, MEES = Mainzer Emergency Evaluation Score

te Unterschiede: So betrug das mediane Patientenalter im Jahr 200366 (MW \pm SD: $61 \pm 23)$ Jahre und $201370(\mathrm{MW} \pm$ SD: $63 \pm 24)$ Jahre $(p<0,01)$. Der Anteil minderjähriger Patienten ( $<18$ Jahre) am Gesamtkollektiv war mit 5,5\% $(n=330)$ und $6,2 \%(n=460)$ vergleichbar, aller- dings kam es zu einem deutlichen Anstieg des Anteils hochbetagter Patienten (Alter $\geq 85$ Jahre) mit einer Verdopplun der Zahl der versorgten Patienten dieser Altersgruppe von $11 \%(n=650)$ au $16 \%(n=1161, p<0,001)$ (ם Abb. 1). Un terschiede in der Geschlechterverteilung konnten nicht nachgewiesen werden; so fanden sich in beiden Kollektiven jeweils $53 \%$ weibliche Patienten.

Erkrankungs- und Verletzungsschwere

Zur Analyse des NACA-Scores konn ten 20035888 Protokolle $(97,6 \%)$ und 20137331 Protokolle $(98,1 \%)$ ausgewertet werden. Im Jahr 2003 wurde bei 498 Protokollen $(8,5 \%)$ und 2013 bei 913 Protokollen (12,5\%) der NACA Score seitens der Autoren während der Datenanalyse anhand der vorliegenden Parameter nachträglich ermittelt. Hierbei ergaben sich jedoch keine wesentlichen Veränderungen im Verteilungsspektrum der einzelnen NACA-Subgruppen. Daher wurden die kalkulierten NACAScores in die Gesamtanalyse inkludiert. Die Analyse der vorliegenden Daten zum NACA-Score ergab eine Änderung hinsichtlich der Häufigkeit lebensbedrohlich erkrankter bzw. verletzter Patienten (NACA IV-VI: $n=829$ [13,8\%] vs. $n=1197[16 \%], p<0,01)$. Zum Bewusstseinszustand nach GCS waren 2003 $5531(91,7 \%)$ und $20136803(91,1 \%)$ Protokolle auswertbar. Auch der Anteil bewusstseinsgetrübter Patienten (GCS < 9) wies mit 476 Patienten in $2003(7,9 \%)$ und 662 Patienten in $2013(8,9 \% ; p=$ $0,04)$ eine Tendenz zu einer höheren Erkrankungsschwere auf. Ebenso wurden nach Berechnung des MEES $1^{*}$ im Jahr 2013 signifikant mehr akut vital gefährdete Patienten behandelt ( $n=850$ $[14,1 \%]$ vs. $n=1184[15,1 \%], p=0,005)$ (a Abb. 2).

\section{Einsatzort}

Zur Auswertung standen 20036024 (99,9\%) und 20137272 (97,3\%) vollständige Angaben zur Verfügung. Im Untersuchungszeitraum zeigte sich ein hochsignifikanter Anstieg der Notarzteinsätze in Pflegeeinrichtungen. So wurden im I. Quartal 2003175 (2,9\%) Patienten in Pflegeeinrichtungen gegenüber 750 (10,3\%) im Jahr 2013 durch den Notarztdienst versorgt $(p<0,01)$. Dementsprechend zeigte sich ein Rückgang der in der eigenen Wohnung behandelten Patienten $(n=4654[77,2 \%]$ vs.

\section{| Der Anaesthesist}




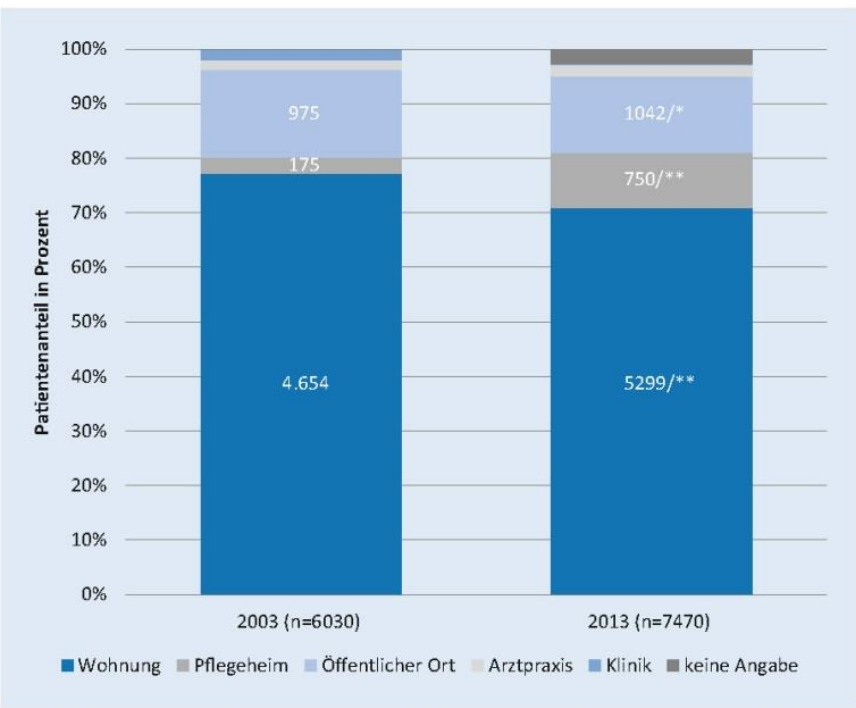

Abb. 3 A Einsatzort der notärztlich versorgten Patienten des I. Quartals 2003 vs. 2013 in Prozent der Einsätze und Absolutzahlen $\left({ }^{*} p<0,05 ;{ }^{*} p<0,001 ; n\right.$ s nicht signifikant)

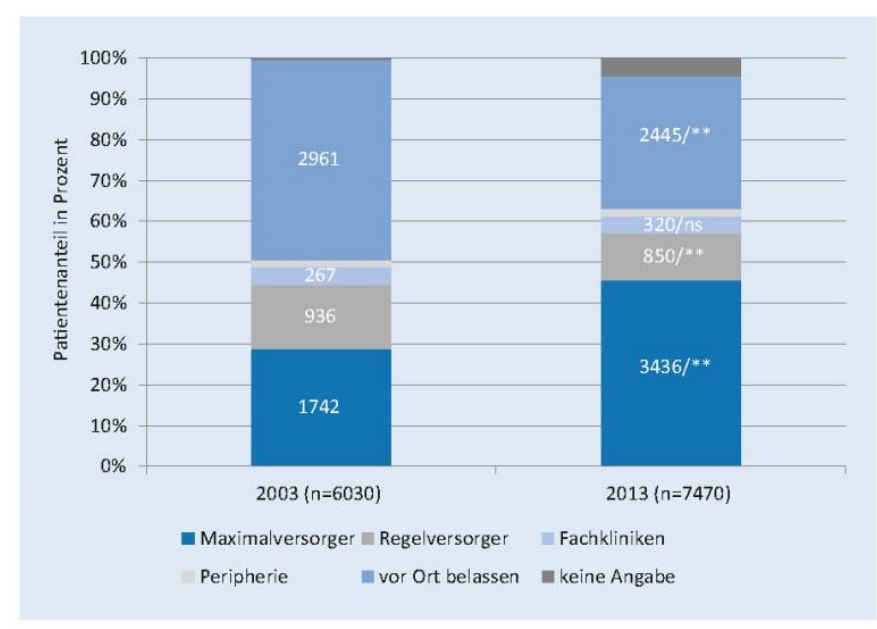

Abb. 4 A Distributionsverhalten der Notärzte des I. Quartals 2003 vs. 2013, Darstellung als Säulendiagramm in Prozent und als Absolutwerte $\left({ }^{* *} p<0,001 ; n s\right.$ nicht signifikant)

$n=5299[72,9 \%], p<0,001)(\mathbf{A b b} .3)$. Signifikant rückläufig war die Anzahl der notärztlich begleiteten interhospitalen Verlegungsfahrten im Stadtgebiet ( $n=$ $116[1,9 \%]$ vs. $n=23[0,3 \%])$.

\section{Einsatzkategorien}

Eine Einsatzkategorisierung anhand der dokumentierten notärztlichen Erstdiagnosen und des Patientenalters war 2003 bei $5758(95,5 \%)$ und 2013 bei 7328 (98,1\%) Patienten möglich. Es zeigt sich sowohl für 2003 als auch für 2013 ein klares Überwiegen internistischer Krankheitsbilder; zusätzlich nahm deren Anteil an der Gesamtheit der Einsätze weiter $\mathrm{zu}(n=3119$ [51,7\%] vs. $n=4298[57,5 \%], p<0,001)$. Auch bei den traumatologischen $(n=296[4,9 \%]$ vs. $n=511[6,8 \%], p<0,001)$ und pädiatrischen/geburtshilflichen Patienten ( $n=334[5,5 \%]$ vs. $n=487$ [6,5\%], $p<$ $0,05)$ ergab sich bei insgesamt deutlich niedrigeren Einsatzzahlen eine signifikante Zunahme der Einsatzhäufigkeit. Weitestgehend konstant zeigte sich der Anteil neurologischer Patienten $(n=675$ [11,2\%] vs, $n=899[12,0 \%], p=0,1$ ) prozentual diskret rückläufig waren hingegen psychiatrische Krankheitsbilder ( $n=752[12,5 \%]$ vs. $n=849[11,4 \%]$, $p<0,05)$.

\section{Transportziel}

Im Jahr 2003 konnten $6010(99,7 \%)$ und 20137183 (96,2\%) der Protokolle zur Auswertung herangezogen werden Der Anteil der Krankenhauszuweisungen nahm im Untersuchungszeitraum $\mathrm{zu}(n=3049[51 \%]$ vs. $n=4738[66 \%]$ $p<0,01)$, der Anteil der vor Ort belassenen Patienten nahm dementsprechend prozentual ab $(n=2961[49 \%]$ vs. $n=$ 2445 [34\%], $p<0,001)$. Bei den der Klinik zugewiesenen Patienten konnten deutliche Unterschiede im notärztlichen Distributionsverhalten zugunsten der Maximalversorger festgestellt werden ( $n=1742$ [29\%] vs. $n=3436[46 \%]$, $p<0,01)$ ) (ם Abb. 4). Der Anteil an Patienten, die Fachkliniken und Krankenhäusern der städtischen Peripherie zugewiesen wurden, blieb konstant ( $n=$ $369[6,1 \%]$ vs. $n=452[6,1 \%], p=$ $0,87)$. Den städtischen Regelversorgern hingegen wurden, trotz gestiegenem Gesamtpatientenaufkommen weniger Patienten zugewiesen $(n=936[15,2 \%]$ vs. $n=850[11,4 \%], p<0,001)$.

\section{Notärztliche Interventionen}

Eine Übersicht der dokumentierten notärztlichen Interventionen ist in $\mathbf{0}$ Tab. 1 dargestellt. Insgesamt erfolgte lediglich bei der Hälfte aller Einsätze eine ärztliche Intervention. Hierbei handelte es 
Originalien

\begin{tabular}{|c|c|c|c|c|c|}
\hline & \multicolumn{2}{|l|}{2003} & \multicolumn{2}{|l|}{2013} & \multirow[t]{2}{*}{$p$} \\
\hline & Anzahln & $\%$ & Anzahln & $\%$ & \\
\hline Venöser Zugang & 3936 & 65,3 & 4016 & 53,8 & $<0,01$ \\
\hline Atemwege freimachen & 98 & 1,6 & 103 & 1,4 & ns \\
\hline Absaugen & 85 & 1,4 & 84 & 1,1 & ns \\
\hline Reanimation & 78 & 1,3 & 95 & 1,3 & ns \\
\hline $\begin{array}{l}\text { NIV/Maske/alternative supraglotti- } \\
\text { sche Atemwegshilfen }\end{array}$ & 70 & 1,2 & 26 & 0,4 & $<0,01$ \\
\hline Verband & 62 & 1,0 & 92 & 1,2 & ns \\
\hline Endotracheale Intubation & 61 & 1,0 & 110 & 1,5 & 0,02 \\
\hline Reposition & 43 & 0,7 & 46 & 0,6 & ns \\
\hline Defibrillation & 41 & 0,7 & 32 & 0,4 & 0,05 \\
\hline Vakuummatratze & 28 & 0,5 & 73 & 1,0 & $<0,01$ \\
\hline Blutstillung & 14 & 0,2 & 6 & 0,1 & 0,02 \\
\hline${ }_{n}$ Stiff neck" & 11 & 0,2 & 69 & 0,9 & $<0,01$ \\
\hline Krisenintervention & 3 & 0,05 & 111 & 1,5 & $<0,01$ \\
\hline Schaufeltrage & 2 & 0,03 & 4 & 0,05 & ns \\
\hline Thoraxdrainage & 2 & 0,03 & 0 & 0,0 & ns \\
\hline Koniotomie & 1 & 0,02 & 2 & 0,03 & ns \\
\hline Entbindung & 0 & 0,0 & 3 & 0,04 & ns \\
\hline
\end{tabular}

\begin{tabular}{|c|c|c|c|c|c|}
\hline & \multicolumn{2}{|l|}{2003} & \multicolumn{2}{|l|}{2013} & \multirow[t]{2}{*}{$p$} \\
\hline & Anzahln & $\%$ & Anzahln & $\%$ & \\
\hline \multicolumn{6}{|c|}{ Unterteilung nach Weiterbildungsstand } \\
\hline Assistenzarzt & 240 & 4,4 & 20 & 0,3 & $<0,01$ \\
\hline Facharzt & 5313 & 95,7 & 6907 & 99,7 & $<0,01$ \\
\hline \multicolumn{6}{|c|}{ Unterteilung nach Fachrichtung } \\
\hline Anästhesie & 1792 & 32,3 & 3562 & 51,4 & $<0,01$ \\
\hline Chirurgie & 875 & 15,8 & 787 & 11,4 & $<0,01$ \\
\hline Innere & 842 & 15,2 & 1470 & 21,2 & $<0,01$ \\
\hline Andere & 2044 & 36,8 & 1108 & 16,0 & $<0,01$ \\
\hline Keine Angabe & 477 & 7,9 & 543 & 7,3 & - \\
\hline
\end{tabular}

sich in der überwiegenden Zahl der Fälle um die Anlage eines periphervenösen Zugangs.

Eine Medikamentengabe erfolgte 2003 signifikant häufiger als im Vergleichszeitraum 2013 (verabreichte Medikamente $n=9085$ vs. $n=8125, p<$ $0,001)$. So wurde 2003 bei $72 \%(n=4351)$ der Patienten die Applikation mindestens eines Medikaments vorgenommen, 2013 wurde nur bei $58 \%(n=4337)$ der Patienten mindestens ein Medikament verabreicht.

Die Anzahl der Lagerungsmaßnahmen nahm im Vergleich beider Beob-

| Der Anaesthesist $n=84, p=0,73$ ); im Jahr 2013 konnten jedoch doppelt so viele Patienten primär erfolgreich reanimiert werden wie 2003 ( $n=15$ vs. $n=31, p=0,08$ ).

\section{Notärztliche Qualifikation}

Zur Beurteilung der notärztlichen Qualifikation konnten 20035553 (92,1\%) und 20136927 (92,7\%) Protokolle herangezogen werden. Im Untersuchungsintervall konnte eine deutliche Zunahme der Facharztquote festgestellt werden. Die Zahl der Notarzteinsätze, die durch Fachärzte für Anästhesiologie $(n=1666$ [30\%] vs. $n=3554[51 \%], p<0,01)$ und Fachärzte für Chirurgie $(n=836$ [15\%] vs. $n=1470[21 \%], p<0,01$ ) versorgtwurden, stieg signifikant an (ם Tab. 2). Der Anteil anderer Fachdisziplinen sowie der Anteil der durch Weiterbildungsassistenten versorgten Patienten nahmen hingegen im Beobachtungszeitraum deutlich ab.

\section{Diskussion}

Ziel der Untersuchung war es, die Entwicklung im bodengebundenen Notarztdienst einer deutschen Großstadt zwischen 2003 und $2013 \mathrm{zu}$ analysieren. Auf Basis der Ergebnisse sollten Möglichkeiten der Ressourcenoptimierung evaluiert und eine bedarfsgerechte Anpassung zukünftiger personeller und infrastruktureller Versorgungskapazitäten möglich werden. Ebenso sollten sich zukünftige Fortbildungs- und Qualifikationsschwerpunkte des am Rettungsdienst beteiligten Personals herauskristallisieren.

achtungszeiträume $\mathrm{zu}(n=41[0,7 \%]$ vs. $n=146[2 \%], p<0,001)$.

Maßnahmen des Atemwegsmanagements wurden 2013 prozentual vergleichsweise seltener als 2003 durch den Notarzt durchgeführt $(n=315[5,2 \%]$ vs. $n=327[4,4 \%], p<0,03)$. Der Anteil der definitiven Atemwegssicherungen mithilfe der endotrachealen Intubation an der Gesamtheit der Maßnahmen des Atemwegsmanagements stieg im Untersuchungszeitraum jedoch an $(n=61$ [46\%] vs. $n=110$ [79\%], $p<0,02$ ).

Der Anteil kardiopulmonaler Reanimationen war in beiden Untersuchungsperioden vergleichbar (je $1 \% ; n=64$ vs.
Im Untersuchungszeitraum konnte ein erheblicher Anstieg der Notarzteinsatzzahlen beobachtet werden. Dabei nahm insbesondere der Anteil geriatrischer Patienten zu. Damit einhergehend war ein deutlicher Anstieg bei Einsätzen in Pflegeheimen zu verzeichnen.

Zudem stieg die Zahl stationärer Einweisungen, wobeieine Konzentration auf Einrichtungen der Maximalversorgung zu beobachten war. Der Anteil therapeutischer Maßnahmen im präklinischen Bereich nahm hingegen ab.

Im Einklang mit vorangegangenen Untersuchungen aus bodengebundenen 
Notarzt- und Luftrettungssystemen [2, 14] zeigt die vorliegende Analyse eine deutliche Steigerung der Notarzteinsatzrate um $21 \%$ und der Notarzteinsatzzahlen um $24 \%$ in einem großstädtischen Versorgungsgebiet über einen Zeitraum von 10 Jahren. Damit verhält sich die Zunahme der Notarzteinsätze nicht proportional zur Entwicklung der Einwohnerzahl im Stadtgebiet Leipzig im entsprechenden Untersuchungszeitraum (Einwohnerzahl 2003 vs. 2013: 497.531 vs. 531.562 , Anstieg: $6,8 \%$ [30]) und lässt sich demnach nicht allein auf einen Bevölkerungszuwachs zurückführen.

Der im bundesdeutschen Durch schnitt 21 \%ige Anstieg der Notarzteinsatzrate im nahezu vergleichbaren Zeitraum ist zwar als gleichwertig anzusehen (2003: 28 Einsätze/1000 Einwohner und Jahr vs. 2013: 34 Einsätze/1000 Einwohner und Jahr $[6,8,29]$, liegt jedoch absolut noch deutlich unter dem hohen Regionalwert für Leipzig. Sowohl im Jahr 2003 als auch im Jahr 2013 war die Leipziger Notarzteinsatzrate demnach jeweils $71 \%$ höher als der bundesdeutsche Durchschnitt. Auch ein Vergleich zu einer Stadt mit ähnlicher Bevölkerungsstruktur und Einwohnerzahl, wie z.B. Hannover, zeigt in Leipzig eine doppelt so hohe Notarzteinsatzrate (Hannover 2012: 26 Einsätze/1000 Einwohner und Jahr [25] vs. Leipzig 2013: 58 Einsätze/1000 Einwohner und Jahr). Ferner zeigt auch die in Hessen in den Jahren 2010/2011 durchgeführte BoLuS-Studie [13] eine geringere Notarzteinsatzrate von im Mittel 28 Einsätzen/1000 Einwohnern und Jahr. Selbst der höchste Maximalwert der dortig betrachteten 12 Studienregionen war mit 42 Einsätzen/1000 Einwohnern und Jahr geringer als derjenige in Leipzig [13].

Bereits in anderen Arbeiten wurde auf den Einfluss des Alters in Bezug auf steigende Notarzteinsatzzahlen hingewiesen $[2,10,17]$. Auch das in der vorliegenden Untersuchung versorgte Patientenkollektiv weist eine deutliche Zunahme älterer und hochbetagter Patienten auf. Der demografische Wandel ist ein gesamtgesellschaftliches und bundesweites Phänomen, das jedoch durch regionale Unterschiede - verursacht durch sozial- und arbeitsmarktpolitisch beding- te Abwanderung und Umsiedlung - gekennzeichnet ist. So ist insbesondere in den östlichen Bundesländern ein deutlicher Anstieg des Anteils der älteren Bevölkerung zu verzeichnen (Gesamtanteil Bevölkerung $>67$ Jahre: Flächenländer West $29,0 \%$ vs. $34,5 \%$ Flächenländer Ost [33]) und betrifft somit auch das Stadtgebiet von Leipzig. Vor dem Hintergrund, dass diese Entwicklung in Städten nicht in gleichem Maße wie auf dem Land stattfindet, muss es offenbar noch andere, die Notarzteinsatzrate beeinflussende Faktoren geben. Auffällig ist hierbei auch der in Leipzig, wenn auch über den Untersuchungszeitraum rückläufige, mit anderen Rettungsdienstbereichen jedoch nichtvergleichbar hohe Anteil zu Hause belassener Patienten. Dies unterstützt die These, dass hier Faktoren wie z. B. optimierbare Notarzteinsatzkriterien, Dispositionsstrategien, aber auch das ungerechtfertigte Übertragen von eigentlich kassenärztlichen Aufgaben an den Notarztdienst eine maßgebliche Rolle bei der Entwicklung der hohen Einsatzzahlen spielen $[12,13]$

Zusätzlich zur deutlichen Zunahme älterer Patienten konnte in der vorliegenden Untersuchung eine signifikante Zunahme der Einsatzhäufigkeit in Pflege- und Seniorenheimen nachgewiesen werden. Dies kann zum einen damit erklärt werden, dass durch die demografische Entwicklung auch deutlich mehr Menschen in Pflegeheimen statt in der eigenen Wohnungleben, zum anderen aber auch, dass Strukturen für die Versorgung von Pflegeheimpatienten wie niedergelassene Årzte und der kassenärztliche Bereitschaftsdienst in immer begrenzterem Maße zur Verfügung stehen.

Notärztliche Weiterbildungsinhalte sollten daher auf die steigende Einsatzhäufigkeit bei multimorbiden und geriatrischen Patienten unter Beachtung medizinischer, ethischer und rechtlicher Besonderheiten (Patientenverfügungen/ Betreuungsverhältnisse) eingehen [16] Ebenso stellt der zunehmende Fachkräftemangel im Gesundheitswesen insbesondere im pflegerischen Bereich, ein zentrales und immer wichtiger werdendes Problemfeld dar $[9,12]$. Eine Reduktion der notärztlichen Einsatz häufigkeit und Steigerung der medi- zinischen Versorgungsqualität älterer Patienten in Pflegeheimen ließe sich z. B. durch Stärkung der für eine nachhaltige Grundversorgung essenziellen pflegerischen Ressourcen, Förderung beruflicher Qualifizierungsmaßnahmen, regelmäßige ärztliche Visitendienste sowie eine stärkere Einbindung des kassenärztlichen Bereitschaftsdienstes erreichen [7]

Durch derartige Maßnahmen ließe sich möglicherweise auch der in unserer Studie beobachtete Anstieg der Nachforderungshäufigkeit des Notarztes durch den nichtärztlichen Rettungsdienst minimieren.

Trotzdeutlich gestiegener Einsatzzahlen und tendenziell angestiegener Erkrankungsschwere hat sich die Häufigkeit spezifischer notärztlicher Maßnahmen im Untersuchungszeitraum in der vorliegenden Untersuchung verringert. Es wurden bei weniger Patienten weniger Medikamente verabreicht (2003: $n=$ 9085 Medikamente bei $n=4351$ Patienten [72\% aller Patienten] vs. $2013: 8125$ Medikamente bei $n=4337$ Patienten [58\% aller Patienten]), und es wurden weniger Maßnahmen des Atemwegsmanagements durchgeführt. Eine mögliche Erklärung hierfür sind bei kurzen innerstädtischen Transportzeiten und der Etablierung zentraler Notaufnahmen ein bewusstes Verschieben von Maßnahmen in die Notaufnahmen, nicht zuletzt, um ggf. auch die Einsatzzeit vor Ort verkürzen zu können und die Patienten, insbesondere bei zeitkritischen Ereignissen (z. B. Apoplex oder Myokardinfarkt), schneller einer definitiven Diagnostik und Therapie zuzuführen $[1,10]$. Aber auch die notärztlich bzw. vor Ort nur begrenzt therapierbaren Krankheitsbilder (z. B. Infektionskrankheiten, Volumen- und Elektrolytstörungen, neurologisch/[geronto-]psychiatrische und chronische Erkrankungen), insbesondere in der Altersgruppe mit der höchsten Fallzahlsteigerung, könnten diesen scheinbaren Widerspruch erklären. Ebenso denkbar ist eine be wusste therapeutische Zurückhaltung aufgrund bestehender Multimorbidität und den damit verbundenen komplexen medikamentösen Therapieregimen (z. B. Wechselwirkungen, Kontraindikationen, Verfügbarkeit) und evtl. auftre- 
Originalien

tenden medikolegalen Problemen der Erreichbarkeit von Angehörigen, der Uneindeutigkeit oder gar des Fehlens einer Patientenverfügung.

Die leichte Zunahme der Erkran kungs- und Verletzungsschwere bei al len Parametern (NACA-Score, GCS, MEES $1^{*}$ ) kann am ehesten auf eine im Rahmen des gestiegenen Alters an zunehmende gesteigerte Gesamt- bzw. Multimorbidität zurückzuführen sein. Aufgrund der bekannten Subjektivität des NACA-Scores und der eingeschränk ten Aussagekraft hinsichtlich eines sich daraus ergebenden innerklinischen Ressourcenbedarfs [3], sind diese Erkenntnisse zwar noch kein Garant für einen Mehrbedarf an innerklinischen Ressourcen (z. B. Schockraum, bildgebend Untersuchungen, Intensivkapazitäten), zeigen jedoch eine Tendenz und sollten daher in zukünftigen transsektoralen Untersuchungen ebenfalls eine zentrale Rolle spielen.

Vor dem Hintergrund der sich verknappenden Ressourcen sowie unte wirtschaftlichen Aspekten gilt es zu erwägen, ob eine konstante, engmaschige ambulante Versorgung, der Ausbau des kassenärztlichen Bereitschaftsdienstes sowie weiterführende Qualifizierungsmaßnahmen insbesondere für das nichtärztliche Rettungsdienstpersonal (Notfallsanitäter) für Patienten mit strittiger Notarztindikation möglicherweise ausreichende und sogar adäquatere, da nachhaltigere, Versorgungsstrategien darstellen könnten [5]. Insbesondere dem nichtärztlichen Rettungsdienstpersonalbzw. der noch relativ jungen Berufs gruppe der Notfallsanitäter kommt hier eine besondere Rolle zu. Es konnte bereits in verschiedenen Arbeiten gezeigt werden, dass entsprechend geschultes und qualifiziertes sowie supervidiertes Personal effektiv und sicher in der Lage ist, ausgewählte ärztliche Funktionen zu übernehmen [15]. Einerseits kann hiermit die prähospitale Behandlungszeit re duziert [19], die Behandlungsqualität fü den Patienten teilweise verbessert (z. B. durch raschere Analgesie und zügigeren Transport zur Definitivversorgung [11]) und auch die „Ressource“ Notarzt für vi tal bedrohte Patienten geschont werden [19]. Ergänzend soll hier auch der Aus- bau telenotfallmedizinischer Strukturen aufgeführt sein, der das nichtärztliche Rettungsdienstfachpersonal vor Ort unterstützen und eine Behandlung ohne Notarzt bis zur Klinikaufnahme bzw. eine Verkürzung des therapiefreien Intervalls bis zum Eintreffen des Notarztes ermöglichen kann [4].

Aus Sicht der Autoren ist die durchgängige Schaffung von integrierten Ret tungsleitstellen mit einer standardisierten undleitsymptomorientierten Notrufabfrage und Einbindung der Disposition vertragsärztlicher Strukturen ein wesentlicher Baustein, um auf die nachweisba re Steigerung der Notarzteinsatzrate zu reagieren und eine fallgerechte Ressourcennutzung zu ermöglichen. Diese An passung wurde im Januar 2016 in Leip zig umgesetzt; hierbei zeigte sich bereit in den ersten 6 Monaten nach Einfüh rung ein Rückgang der Notarzteinsatzhäufigkeit um $21 \%$ [Daten NEF Standort Leipzig Mitte 01-06/16; Branddirektion Leipzig, 2016]. Allerdings zeigt sich dem entsprechend eine Steigerung der rettungsdienstlich versorgten Patientenzahlen, sodass weitere Untersuchungen hier$\mathrm{zu}$ folgen müssen.

Des Weiteren könnten nach ärztlicher Beratung bzw. unter Berücksichtigung etablierter Alarmierungsstandards un Vorgaben Anrufe mit geringer bis keine dringlichen Indikation an den vertrags ärztlichen Notdienst weitergeleitet oder durch eine telefonische Beratung ausreichend versorgt werden. Ergänzend dürf te die Etablierung von Notfallpraxen mit ganztägiger Einsatzbereitschaft zu eine weiteren Entlastung der notfallmedizinischen Strukturen führen $[5,26]$.

Bezüglich der Einsatzgründe haben sich in der vorliegenden Untersuchung nur geringfügige Verschiebungen hinsichtlich der Häufigkeiten ergeben; maß geblich bleiben internistische, traumato logische, aberauch sozial-/psychiatrische Alarmierungsgründe am häufigsten vertreten. Vor dem Hintergrund einer notwendig erscheinenden Ressourcenop timierung ist hier insbesondere der Bereich der sozial-/psychiatrischen Einsatzgründe im Notarztdienst kritisch zu hinterfragen [28]. Ein niedrigschwelliger Zugang $\mathrm{zu}$ alternativen Versorgungsmöglichkeiten (z. B. Zuweisung zu psychiatrischen Ambulanzen, Suchtberatungs- und Betreuungseinrichtungen, Kriseninterventionsdiensten, Opferberatungsstellen) birgt ein zusätzliches Entlastungspotenzial für prä- und in nerklinische Versorgungsstrukturen und sichert eine adäquatere, weil wiederum nachhaltigere Versorgung jener Patientengruppe.

Bei der Betrachtung der Notarztqualifikation fällt eine Steigerung der Facharztquote auf, die vermeintlich auf eine bessere Qualität der medizinischen Versorgung hinweisen sollte, dennoch wurde im Rahmen der Untersuchung eine deutliche Zunahme der Krankenhauseinweisungen/-vorstellungen und ein damit verbundener geringerer VorOrt-Verbleib der Patienten nachgewiesen. Gründe hierfür könnten wiederum im erhöhten Patientenalter und der damit verbundenen zunehmenden Multimorbidität, der Immobilität und den damit assoziierten häuslichen Versorgungsproblemen liegen [12, 24].

In der vorliegenden Untersuchung, und nach Kenntnis der Autoren erstmalig, ließ sich zudem eine deutliche Veränderung im Distributionsverhalten der Notärzte zugunsten von Krankenhäusern der Maximalversorgung nachweisen. Einige Regelversorger der Region haben hierbei z. T. proportional zum generellen Anstieg des Patientenaufkommens mehr Patienten zugewiesen bekommen, an deren wurden sogar - trotz gestiegenen Gesamtaufkommens - weniger Patienten zugewiesen. Auffällig ist hierbei, dass die Kliniken der Maximalversorgung doppelt so viele Patienten erhielten. Vor dem Hintergrund dieser Daten lässt sich das häufig wahrgenommene „overcrowding “ in den entsprechenden Notaufnahmen nachvollziehen [27, 34].

Die Hintergründe für die veränderte Zuweisungspraxis im Notarztdienst können aufgrund fehlender innerklinischer Verlaufsdaten nur spekulativ bleiben. Ursächlich spielen in diesem Zusammenhang abermals die zunehmende Multimorbidität und das häufig daraus resultierende Erfordernis eines interdisziplinären Behandlungsansatzes eine wesentliche Rolle. Demzufolge bedürfen Krankenhäuser und im Speziellen deren Notfallversorgungsstrukturen mit einem 
derart nachweisbaren Patientenzuwachs auch einer bedarfsorientierten Anpassung personeller und infrastruktureller Kapazitäten.

Ferner sollte das Konzept der zentralen, interdisziplinären Notaufnahme auch an Standorten der Grund- und Regelversorgung zur Anwendung kommen. So könnte dann, beispielsweise mithilfe einer zentralen/EDV-gesteuerten Patientenverteilung durch di Leitstelle, eine effizientere Zuweisung und Weiterbehandlung der Patienten unter optimaler Nutzung aktuell verfügbarer struktureller Ressourcen (z. B. Intensiv-/Bettenkapazitäten, Personal, Equipment) ermöglicht werden [34] In diesem Zusammenhang wäre die Einbindung ambulanter Versorgungs strukturen in die Disposition durch die Leitstelle zu diskutieren.

$\mathrm{Zu}$ einer weiteren Entlastung innerklinischer Strukturen würde eine Verbes serung des Datenaustausches zwischen den Notfallzentren sowie eine einheitliche, vom Patienten mitzuführende Anamnese-/Medikamentendokumentation, wie beispielsweise in Form der viel diskutierten elektronischen Gesund heitskarte, beitragen.

Um den vorgenannten Problemen und Veränderungen gerecht $\mathrm{zu}$ werden, sollte sowohl in der präklinischen Notfallversorgung als auch im Schnittstellenbereich der Notaufnahme ein zentrales Qualitätsmanagement etabliert werden, dessen Basis die kombinierte Auswertung von prä- und innerklinischen Daten darstellt [12]. Hierfür is eine lückenlose und (mobil-)elektronische Datenerhebung unerlässlich. Denn wie auch bei Auswertung dieser Arbeit und mehrfach in der Literatur kritisiert, stellt die mangelnde Dokumentations qualität oft ein Hindernis im Treffen belastbarer und vergleichbarer Aussagen dar. Vor diesem Hintergrund und um folgenden wissenschaftlichen Arbeiten eine bessere und vergleichbarere Daten basis zu liefern, muss die notärztliche Dokumentation auch an moderne notfallmedizinische, $z$. T. bisher nur innerklinisch angewandte, Dokumentations- $/$ Bewertungskriterien wie $z$. B. eine Angabe von Tracer-Diagnosen anknüpfen [10].

\section{Limitation}

Die vorliegende Untersuchung wird durch das retrospektive Studiendesign und den gewählten 10-jährigen Zeitraum in der Aussagekraft eingeschränkt. Eine weiter zurückgreifende Analyse war jedoch vor dem Hintergrund der fehlenden Archivierungspflicht ältere Protokolle limitiert. Hinsichtlich des Gesamteinsatzaufkommens wurde die Studie von Beginn an auf bodengebundene Notarzteinsätze limitiert, und Luftrettungsdaten waren nicht verfügbar. Darüber hinaus kann die Auswahl des analysierten I. Quartals in den Jahren 2003 und 2013 Einfluss auf die Ergebnisse haben, da beispielsweise in den Sommermonaten ein anderes Einsatzspektrum als im Frühjahr vorliegen könnte. In sich besteht aber eine Vergleichbarkeit vor dem Hintergrund der Auswertung der gleichen Jahreszeit im 10-jährigen Abstand.

Des Weiteren wird die Qualität der Datenerhebung durch teilweise erheb liche Defizite in der einsatzbezogenen Dokumentation beeinträchtigt. Einzelne Parameter (z. B. NACA-Score) unterlie gen zudem einem ausgeprägten subjektiven Bias in Bezug auf die Beurteilung der Erkrankungs-/Verletzungsschwere und sind - auch unter Einbeziehung alternativer Scoring-Systeme wie GCS ode MEES zur Nivellierung der Subjektivitä - nur eingeschränkt verwertbar.

Wesentliche Erkenntnisse aus voran gegangenen Untersuchungen konnten jedoch reproduziert werden $[2,14]$, sodass die diskutierten Ergebnisse reliabel erscheinen.

\section{Fazit für die Praxis}

- Eine im Vergleich zum Bundesdurchschnitt $71 \%$ höhere Notarzteinsatzrate geht im Leipziger Stadtgebiet mit deutlich steigenden Notarzteinsatzzahlen einher.

- Das notärztlich versorgte Patientenkollektiv beinhaltet einen zunehmenden Anteil hochbetagter sowie in Pflegeeinrichtungen untergebrachter Patienten.

- Einhergehend mit diesen Beobachtungen finden sich ein Anstieg der
Krankenhauszuweisungen und eine Änderung im Distributionsverhalten zugunsten Kliniken der Maximalversorgung.

- Optimierungspotenzial besteht hinsichtlich der Disposition durch die Rettungsleitstelle, Qualifizierungsmaßnahmen im ärztlichen und nichtärztlichen Bereich sowie der Nutzung alternativer Versorgungsstrukturen.

- Grundlage einer erforderlichen Ressourcenoptimierung sollte eine lückenlose, elektronische Datenerhebung als Basis für ein suffizientes Qualitätsmanagement unter Einbeziehung transsektoral erfasster präsowie innerklinischer Daten sein.

\section{Korrespondenzadresse \\ PD Dr. med. M. Bernhard \\ Zentrale Notaufnahme, Universitätsklinikum Leipzig \\ Liebigstraße 20,04103 Leipzig, Deutschland michael.bernhard@medizin.uni-leipzig.de}

\section{Einhaltung ethischer Richtlinien}

Interessenkonflikt. K. Bader, M. Bernhard, A. Gries M. Kaul, R. Schröder und A. Ramshom-Zimmer geben an, dass kein Interessenkonflikt besteht.

Dieser Beitrag beinhaltet keinevon den Autoren durchgeführten Studien an Menschen oder Tieren.

\section{Literatur}

1. Alberts MJ, Perry A, Dawson DVet al (1992) Effects of public and professional education on reducing
the delay in presentation and referral of stroke patients. Stroke 23:352-356

2. Bernhard M, Hilger T, Sikinger $M$ et al (2006)

. Bernhard $M$, Hilger $T$, Sikinger $M$ et al (2006) Patientenspektrum im Notarztdienst: Was hat sich in den letzten 20 Jahren geändert? Anaesthesist 55:1157-116

3. Bernhard M, Trautwein S, Stepan R, Zahn P, Greim CA, Gries A (2014) Notärztliche Einschätzung der klinischen Weiterversorgung von Notfallpatienten. Anaesthesist 63:394-400

4. Brokmann JC, Rossaint R, Hirsch F et al (2016) Analgesia by telemedically supported paramedics compared with physician-administered analgesia: A prospective, interventional, multicentre trial. Eur JPain 20:1176-1184

5. Brokmann JC, DönitzS, Bischoni Ret al (2013) Notfallsanitätergesetz.NotfRettungsmed 16:604-610 6. Bundesanstalt für Straßenwesen (2015) Leistungen des Rettungsdienstes 2012/13. Unterreihe Menschund Sicherheit, Heft M260.

7. Burgkhardt A (2007) Betrachtung zur Implementierung eines Dringlichen Hausbesuchsdienstes (DHD) als Element der ärztlichen Dringlichkeits- 


\section{Originalien}

versorgung und der Einfluss auf die Senkung der Notarzteinsatzrate Master-Thesis DonauUniversitätKrems

8. Burgkhardt M (2012) Mehr Rettungseinsätze - längere Eintreffzeiten Pressemilleilung der - langere Eintreffzeiten, Pressemitteilung der NotärzteDeutschlands (BAND)

9. Dreier A, RogalskiH(2015) Erwartungen, Wünsch Dreier A RogalskiH(2015) Erwartungen, Wüns und Grenzen der künftigen Aufgabenteilung
von Pflege und Medizin - Ergebnisse. Pflege 28:287-296

10. Fachexperten der Eckpunktepapier-KonsensusGruppe, Fischer $M$, Kehrberger $E$, Marung $\mathrm{H}$ et al (2016) Eckpunktepapier 2016 zur notfallmedizinischen Versorgung der Bevölkerung in der Prähospitalphase und in der Klinik. Notf Rettungsmed 19:387-395

11. Grebl, WranzeE, HartmannH Het al (2011) Analgesi beim Extremitätentrauma durch Rettungsfachpersonal. NotfRettungsmed 14:135-142

12. Gries $A$, Bernhard $M$, Helm M, Brokmann J, Graesner JT (2017) Zukunft der Notfallmedizin in Deutschland 2.0. Anaesthesist 66:307

13. Gries A, Lenz W, Stahl P, Spiess R, Luiz T, (BoLuSStudiengruppe) (2014) räklinische Versorgungszeiten bei Einsätzen der Luftrettung - Einfluss der Dispositionsstrategie der Rettungsleitstelle Anaesthesist 63:555-562

14. Gries A, Zink W, Bernhard $M$, Messelken $M$, Schlechtriemen $T(2006)$ Realistic assesment of the physician-staffed emergency services in Germany. Anaesthesist 55:1080-1086

15. Häske D, Schmepf $B_{i}$ Gaier $G$, Niederberge C (2014) Prähospitale Analgosedierung durch Rettungsassistenten. Anaesthesist63:209-216

16. Heindl P, Ruppert S, Kozon V (2014) Experiences of Austrian nursing staff in dealing with advance of Austrian nursing staff in dea
directives. Pflege 27:337-346

17. Hennes HJ, Reinhardt T, Dick W (1992) Beurteilung des Notfallpatienten mit dem Mainz EmergencyEvaluation Score MEES. Notfallmedizin 18:130-136

18. Heppner HJ, Wiesner R, Schuster S et al (2014) Bedeutung der demographischen Entwicklung für die Notfallmedizin. NotfRettungsmed 17:494-499

19. Kill C, Greb I, Wranze E et al (2007) Kompetenzentwicklung im Rettungsdienst. Notf Rettungsme 10:266-272

20. Köller M (2014) Geriatrische Aspekte bei betagten Notfallpatienten. NotfRettungsmed 17:484-487 1. Korzilius H, Rieser S, Schulte Strathaus R (2015) Ambulante Notfallversorgung: Patienten behandeln - aber am richtigen Ort. Dtsch Arztebl 112:A353-A356

22. Mochmann HC, Arnzt HR, Bobbert P (2014) Der Notarzt im Altenheim - Vorurteil und Wirklichkeit. NotfRettungsmed 17:131-136

23. Plass D, Vos T, Hornberg Cet al (2014) Entwicklun der Krankheitslast in Deutschland. Ergebnisse, Potenziale und Grenzen der Global Burden of Potenziale und Grenzen der Global Burden 24. Prückner S, Luiz T, Steinbach-Nordmann S (2008) Notfallmedizin - Medizin für eine alternde Notfallmedizin - Medizin für eine al

Gesellschaft Anaesthesist57:391-396
25. Rettungsdienstbedarfsplan für die Landeshaupt25. Rettungsdienstbedarfsplan für die Landeshaup
stadt Hannover Fortschreibung (2013) https:// stadt Hannover Fortschreibung (2013) https:// egovernment.hannoverstadt.de/hhSIMwebd/. nsf/99730F67E775F515C1257C0B004096BD/ SFILE/2280-201

26. Riessen R, Gries A, Seekamp A et al (2015) Positionspapier für eine Reform dermedizinischen Notfallversorgung in deutschen Notaufnahmen. NotfRettungsmed 18:174-185

\section{Der Anaesthesist}

27. Searle J, Muller R Slagman A et al (2015) Öberfüllung der Notaufnahmen. Gründe und populationsbezogene Einflussfaktoren. Notf Rettungsmed 18:306-315

28. Seiger K, Brommundt J (2002) Krisenintervention im Rettungsdienst - eine Herausforderung an den Notarzt? NotfRettungsmed 5:116-118

29. Sikinger $M$, Bernhard $M$, Bujard $M$ et al (2005) . Siknger $M_{2}$ Bernhard $M_{4}$ Bujard $M$ et al (2005) Notfallmedizin gestern, heute und morgen
-40 Jahre Notarztstandort Heidelberg. Notf Rettungsmed 8:133-137

30. Stadt Leipzig (2015) Informationssystem, Bevölkerungsentwicklung. http://statistik.leipzig.de/ statcity/table.aspx?cat $=2 \&$ rub $=1 \& o b j=0$. Zugegriffen:9. Febr. 2015

31. Statistische Ämter des Bundes und der Länder (2011) Statistischer Bericht Al3/111, Berlin Population 2011. https://www.statistik-berlinbrandenburg.de/Publikationen/Aufsaetze/2014/ HZ_201403-01.pdf.Zugegriffen: 25.03.2017

32. Statistische Ämter des Bundes und der Länder (2011) Demografischer Wandel in Deutschland, Heft 1. https://www.destatis.de/ DE/Publikationen/Thematisch/Bevoelkerung/ DemografischerWandel/ BevoelkerungsHaushaltsentwicklung.html. Zugegriffen: 25.03.2017

33. Statistisches Bundesamt (2015) Bevölkerungsentwicklung in den Bundesländern bis 2060, Ergebnisse der 13. koordinierten Bevölkerungsvorausberechnung

34. Zimmermann M, Brokmann $J C$, Gräff I et al (2016) Zentrale Notaufnahme - Update 2016. Anaesthesist 65:243-249 


\title{
3. Zusammenfassung der Arbeit
}

\author{
Publikationspromotion zur Erlangung des akademischen Grades \\ Dr. med.
}

\section{Entwicklung bodengebundener Notarzteinsätze im Stadtgebiet Leipzig von 2003 bis 2013}

eingereicht von Katja Bader, geb. Jung angefertigt am Universitätsklinikum Leipzig, Zentrale Notaufnahme betreut von Professor Dr. med. André Gries, Privatdozent Dr. med. habil. Michael Bernhard, Dr. med. Alexandra Ramshorn-Zimmer

Februar 2018

Die vorliegende retrospektive Analyse wertet Notarzteinsätze des Stadtgebietes Leipzig im Jahr 2003 und 2013 aus und vergleicht die erhobenen Daten miteinander, um Rückschlüsse auf Entwicklungen des Notarztdienstes ziehen zu können. Auf Basis der Ergebnisse sollten Möglichkeiten der Ressourcenoptimierung evaluiert und eine bedarfsgerechte Anpassung zukünftiger personeller und infrastruktureller Versorgungskapazitäten ermöglicht werden. Ebenso sollten sich Schwerpunkte für zukünftige Fortbildungs- und Qualifikationsangebote für das am Rettungsdienst beteiligte Personal herauskristallisieren.

Im deutschen Rettungsdienst wird über die kontinuierlich zunehmende Zahl von Notarzteinsätzen berichtet. Diese steigen stärker an, als noch vor wenigen Jahren 
prognostiziert. Bisher lagen jedoch kaum Daten aus Großstädten vor, die diese Veränderungen über einen längeren Zeitraum darstellten.

In der retrospektiven Untersuchung wurden die Notarzteinsätze jeweils des Zeitraums 01. Januar bis 31. März der Jahre 2003 und 2013 der Stadt Leipzig ausgewertet. Neben demografischen Patientendaten (Alter, Geschlecht) wurde die Notarzteinsatzrate (Notarzteinsätze/1000 Einwohner und Jahr), die Erkrankungs- und Verletzungsschwere (National Advisory Committee of Aeronautics [NACA]-Score), der Einsatzort und der Versorgungsgrad der Zielklink erfasst.

Im Untersuchungszeitraum konnte ein erheblicher Anstieg der Notarzteinsatzzahlen im Stadtgebiet Leipzig beobachtet werden. Diese nahmen in den korrespondierenden Zeiträumen um $24 \%$ zu (6030 vs. 7470 ), die Notarzteinsatzrate stieg hierbei um $21 \%$. Damit verhält sich die Zunahme der Notarzteinsätze nicht proportional zur Entwicklung der Einwohnerzahl im Stadtgebiet Leipzig im Vergleichszeitraum (Einwohnerzahl 2003 vs. 2013: 497.531 vs. 531.562, Anstieg: 6,8\%) und lässt sich demnach nicht allein auf den Bevölkerungszuwachs zurückführen.

Das Patientenalter betrug im Jahr 2003 im Median 66 und 2013 im Median 70 Jahre, hierbei zeigt sich nahezu eine Verdopplung des geriatrischen Patientengutes im Alter $\geq 85$ Jahre (650 vs. 1161). Dementsprechend vervierfachte sich die Anzahl der Einsätze in Pflegeheimen (175 vs. 750). Dies kann zum einen damit erklärt werden, dass durch die demografische Entwicklung auch deutlich mehr Menschen in Pflegeheimen statt in der eigenen Wohnung leben, zum anderen aber auch, dass Strukturen für die Versorgung von Pflegeheimpatienten wie niedergelassene Ärzte und der kassenärztliche Bereitschaftsdienst in immer begrenzterem Maße zur Verfügung stehen. Notärztliche Weiterbildungsinhalte sollten daher auf die steigende Einsatzhäufigkeit bei multimorbiden und geriatrischen Patienten unter Beachtung medizinischer, ethischer und rechtlicher Besonderheiten (Patientenverfügungen/ Betreuungsverhältnisse) eingehen.

Es wurden im Jahr 2013 deutlich häufiger lebensbedrohlich erkrankte bzw. verletzte Patienten behandelt (NACA IV-VI: 811 vs. 1078; 13,8\% vs. 16,8\%). Trotz vergleichbarem Anteil der Reanimationen am Gesamteinsatzaufkommen (1,3\%), konnten 2013 mehr Patienten primär erfolgreich reanimiert werden (15 vs. $31 ; 0,3 \%$ vs. $0,4 \%$ ). Zudem verdoppelte sich die Zahl der endotrachealen Intubationen ( 61 vs. $110 ; 46,2 \%$ vs. $78,6 \%$ ). 
Insgesamt war jedoch eine Abnahme des Anteils therapeutischer Maßnahmen (insbesondere die Verabreichung von Medikamenten) im präklinischen Bereich zu beobachten.

Im Untersuchungszeitraum stieg nicht nur die Zahl der eingewiesenen Patienten, sondern es konnten auch Unterschiede im notärztlichen Distributionsverhalten hinsichtlich der Krankenhauseinweisungen festgestellt werden (3049 vs. $4738 ; 51 \%$ vs. $66 \%$ ). Dabei wurden vorwiegend die maximalversorgenden Kliniken im Stadtgebiet frequentiert. Hier kam es zu einer Verdopplung der Zuweisungszahlen, wohingegen Krankenhäuser der Regelversorgung einen Rückgang der rettungsdienstlich zugewiesenen Patientenzahlen zeigten. Nach Kenntnis der Autoren wurde diese Entwicklung erstmalig in dieser Form und diesem Ausmaß nachgewiesen. Angesichts der hier dargestellten Daten lässt sich das häufig wahrgenommene „overcrowding“ in den entsprechenden Notaufnahmen nachvollziehen.

Die Ursachen für die veränderte Zuweisungspraxis im Notarztdienst können aufgrund fehlender innerklinischer Verlaufsdaten nur spekulativ bleiben. Mutmaßlich spielen in diesem Zusammenhang abermals die zunehmende Multimorbidität und die häufig daraus resultierende Erfordernis eines interdisziplinären Behandlungsansatzes eine entscheidende Rolle. Angesichts dieser Herausforderungen sollte das Konzept einer zentralen, interdisziplinären Notaufnahme auch an Standorten der Grund- und Regelversorgung zur Anwendung kommen.

Zudem könnte mittels einer zentralen, EDV-gesteuerten Patientenverteilung durch die Leitstelle, eine effizientere Zuweisung und Weiterbehandlung der Patienten unter optimaler Nutzung aktuell verfügbarer struktureller Ressourcen (z. B. Intensiv-/Bettenkapazitäten, Personal, Equipment) ermöglicht werden. In diesem Zusammenhang wäre die Einbindung ambulanter Versorgungsstrukturen in die Disposition durch die Leitstelle ebenso zu diskutieren.

Zusammenfassend zeigt die Untersuchung, dass das Gesamtaufkommen von Notarzteinsätzen weiterhin rapide steigt und der Anteil schwer erkrankter und geriatrischer Patienten zunimmt. Aus Sicht der Autoren ist die durchgängige Schaffung von integrierten Rettungsleitstellen mit einer standardisierten und leitsymptomorientierten Notrufabfrage und die unbedingte Einbindung der Disposition vertragsärztlicher Strukturen ein wesentlicher Baustein, um auf die nachweisbare Steigerung der Notarzteinsatzrate und die 
auffallend hohe, um 71\% über dem Bundesdurschnitt liegende, lokale Notarzteinsatzrate zu reagieren und eine fallgerechte Ressourcennutzung zu ermöglichen. Ergänzend dürfte die Etablierung von Notfallpraxen mit ganztägiger Einsatzbereitschaft zu einer weiteren Entlastung der notfallmedizinischen Strukturen führen.

Grundlage der erforderlichen Ressourcenoptimierung sollte eine lückenlose, elektronische Datenerhebung Grundlage für ein suffizientes, zentrales Qualitätsmanagement - sowohl in der präklinischen Notfallversorgung, als auch im Schnittstellenbereich der Notaufnahme unter Einbeziehung transsektoral erfasster prä- sowie innerklinischer Daten sein. 


\section{Literaturverzeichnis}

Folgende Literaturstellen wurden in der Originalpublikation und in der vorliegenden Promotionspublikation zitiert (in alphabetischer Reihenfolge):

1 Ahnefeld, F (1994) Rettungsdienst und Notfallmedizin: Rück- und Ausblick. Rettungsdienst 17, S. 88- 93

2 Alberts, Mark J.; Perry, April; Dawson, Deborah V. et al (1992) Effects of public and professional education on reducing the delay in presentation and referral of stroke patients. Stroke.23: 352-356

3 Bernhard $M$, Hilger $T$, Sikinger $M$, et al. (2006) Patientenspektrum im Notarztdienst: Was hat sich in den letzten 20 Jahren geändert? Anaesthesist 55: 1157-1165

4 Bernhard M, Trautwein S, Stepan R, Zahn P, Greim C-A, Gries A (2014) Notärztliche Einschätzung der klinischen Weiterversorgung von Notfallpatienten. Anaesthesist 63:394-400

5 Biege, B (1994) Luftrettung: Geschichte, Technik, Organisation. Stumpf \& Kossendey, ISBN-10: 3923124511

6 Blank, I (2/2003) Vom Samariter zum Notarzt. ADAC Luftrettung

7 Brokmann JC, Dönitz S, Bischoni R et al. (2013) Notfallsanitätergesetz. Notfall Rettungsmed 16: 604-610

8 Brokmann JC, Rossaint R, Hirsch F, et al. (2016) Analgesia by telemedically supported paramedics compared with physician-administered analgesia: A prospective, interventional, multicentre trial. Eur J Pain 20: 1176-1184

9 Bundesanstalt für Straßenwesen (2015) Leistungen des Rettungsdienstes 2012/13. Unterreihe Mensch und Sicherheit, Heft M260

10 Burgkhardt A (2007) Betrachtung zur Implementierung eines Dringlichen Hausbesuchsdienstes (DHD) als Element der ärztlichen Dringlichkeitsversorgung 
und der Einfluss auf die Senkung der Notarzteinsatzrate, Master-Thesis, DonauUniversität Krems

11 Burgkhardt M (2012); Mehr Rettungseinsätze - längere Eintreffzeiten, Pressemitteilung der Bundesvereinigung der Arbeitsgemeinschaften Notärzte Deutschlands (BAND) e.V.

12 Dreier A, Rogalski H (2015); Erwartungen, Wünsche und Grenzen der künftigen Aufgabenteilung von Pflege und Medizin - Ergebnisse; Pflege 28: 287-296

13 Fischer $\mathrm{M}$, Kehrberger $\mathrm{E}$, Marung $\mathrm{H}$, et al. und Fachexperten der EckpunktepapierKonsensus-Gruppe (2016) Eckpunktepapier 2016 zur notfallmedizinischen Versorgung der Bevölkerung in der Prähospitalphase und in der Klinik. Notfall Rettungsmed 19: 387-395

14 Gauger, JF (2011) Eine kritische Betrachtung der Entwicklungen im deutschen Rettungswesen. Bachelor-Thesis. Karlsruhe, Karlsruher Institut für Technologie, Institut für Operations Research

15 Gittner, B (1970), Lebenserinnerungen eines Leipziger Kassenarztes v. 1923-1970, Handschriftliche Aufzeichnungen

16 Greb I, Wranze E, Hartmann H, et al. (2011) Analgesie beim Extremitätentrauma durch Rettungsfachpersonal. Notfall Rettungsmed 14: 135-142

17 Gries A, Bernhard M, Helm M, Brokmann J, Graesner JT (2017) Zukunft der Notfallmedizin in Deutschland 2.0. Anaesthesist 66: 307

18 Gries A, Lenz W, Stahl P, Spiess R, Luiz T (BoLuS-Studiengruppe) (2014) Präklinische Versorgungszeiten bei Einsätzen der Luftrettung - Einfluss der Dispositionsstrategie der Rettungsleitstelle. Anaesthesist 63: 555-562

19 Gries A, Zink W, Bernhard M, Messelken M, Schlechtriemen T (2006) Realistic assesment of the physician-staffed emergency services in Germany. Anaesthesist 55: $1080-1086$

20 Häske D, Schmepf B, Gaier G, Niederberger C (2014) Prähospitale Analgosedierung durch Rettungsassistenten. Anaesthesist 63: 209-216

21 Heindl P, Ruppert S, Kozon V (2014); Experiences of Austrian nursing staff in dealing with advance directives; Pflege 27: 337-46 
22 Hellmich, C (2010) Qualitätsmanagement und Zertifizierung im Rettungsdienst. Grundlagen - Techniken - Modelle - Umsetzung. Springer, Heidelberg, Berlin

23 Hennes HJ, Reinhardt T, Dick W (1992) Beurteilung des Notfallpatienten mit dem Mainz Emergency Evaluation Score MEES. Notfallmedizin 18: 130-136

24 Heppner HJ, Wiesner R, Schuster S, et al. (2014) Bedeutung der demographischen Entwicklung für die Notfallmedizin. Notfall Rettungsmed 17: 494-499

25 Kill C, Greb I, Wranze E, et al. (2007) Kompetenzentwicklung im Rettungsdienst. Notfall Rettungsmed 10: 266-272

26 Kirschner, M (1938) Der Verkehrsunfall und seine erste Behandlung. Langenbecks Archiv klinischer Chirurgie 193, 230-302

27 Köller M (2014) geriatrische Aspekte bei betagten Notfallpatienten. Notfall Rettungsmed 17: 484-487

28 Korzilius H, Rieser S, Schulte Strathaus R (2015) Ambulante Notfallversorgung: Patienten behandeln - aber am richtigen Ort. Dtsch Arztbl 112: A353-A356

29 Lay, A (2002): Auswertung der Notarzteinsätze in Bayern auf dem DIVI- Protokolle als Basis für ein präklinisches Qualitätsmanagement, Dissertation am Institut für Anaesthesiologie der Universität Würzburg

30 Menhorn, V (2008): Die Leitlinien müssen eingehalten werden, Dtsch Arztebl 105, S. 1956)

31 Messelken, M (2001): Zentrale Auswertung von Notarzteinsätzen im Rahmen externer Qualitäts-sicherung, Notfall Rettungsmed., S. 408ff.

32 Mochmann HC, Arnzt HR, Bobbert P (2014) Der Notarzt im Altenheim - Vorurteil und Wirklichkeit. Notfall Rettungsmed 17: 131-136

33 Plass D, Vos T, Hornberg C, et al. (2014) Entwicklung der Krankheitslast in Deutschland. Ergebnisse, Potenziale und Grenzen der Global Burden of DiseaseStudie. Dtsch Arztebl Int 111: 629-638

34 Prückner S, Luiz T, Steinbach-Nordmann S (2008); Notfallmedizin - Medizin für eine alternde Gesellschaft; Anaesthesist 57:391-396

35 Rettungsdienstbedarfsplan für die Landeshauptstadt Hannover Fortschreibung 2013; https://egovernment.hannoverstadt.de/lhhSIMwebdd.nsf/99730F67E775F5 15C1257C0B004096BD/\$FILE/2280-2013_Anlage1.pdf 
36 Riessen R, Gries A, Seekamp A, et al. (2015) Positionspapier für eine Reform der medizinischen Notfallversorgung in deutschen Notaufnahmen. Notfall Rettungsmed 18: 174-185

37 Searle J, Muller R, Slagman A, et al. (2015) Überfüllung der Notaufnahmen. Gründe und populationsbezogene Einflussfaktoren. Notfall Rettungsmed 18: 306315

38 Sefrin, P (2003) Geschichte der Notfallmedizin in Deutschland - unter besonderer Berücksichtigung des Notarztdienstes. AINS 38, 623-629

39 Seiger K, Brommundt J (2002), Krisenintervention im Rettungsdienst - eine Herausforderung an den Notarzt? Notfall \& Rettungsmed 5: 116-18

40 Sikinger M, Bernhard M, Bujard M, et al. (2005) Notfallmedizin gestern, heute und morgen - 40 Jahre Notarztstandort Heidelberg. Notfall Rettungsmed 8: 133137

41 Stadt Leipzig, Informationssystem, Bevölkerungsentwicklung; zuletzt geprüft am 09.02.2015.; http://statistik.leipzig.de/statcity/table.aspx?cat=2\&rub=1\&obj=0

42 Statistische Ämter des Bundes und der Länder (2011) Statistischer Bericht Al3/j11, Berlin Population 2011. https://www.statistikberlinbrandenburg.de/Publikationen/Aufsaetze/2014/HZ_201403-01.pdf Zugegriffen: 25.03.2017

43 Statistische Ämter des Bundes und der Länder (2011), Demografischer Wandel in Deutschland,Heft1;https://www.destatis.de/DE/Publikationen/Thematisch/Bevo elkerung/DemografischerWandel/BevoelkerungsHaushaltsentwicklung.html

44 Statistisches Bundesamt, Bevölkerungsentwicklung in den Bundesländern bis 2060, Ergebnisse der 13. koordinierten Bevölkerungsvorausberechnung; 2015

45 Zimmermann M, Brokmann JC, Gräff I, et al. (2016) Zentrale Notaufnahme Update 2016. Anaesthesist 65: 243-249 


\section{Erklärung über die eigenständige Abfassung der Arbeit}

Hiermit erkläre ich, dass ich die vorliegende Arbeit selbstständig und ohne unzulässige Hilfe oder Benutzung anderer als der angegebenen Hilfsmittel angefertigt habe.

Ich versichere, dass Dritte von mir weder unmittelbar noch mittelbar eine Vergütung oder geldwerte Leistungen für Arbeiten erhalten haben, die im Zusammenhang mit dem Inhalt der vorgelegten Dissertation stehen, und dass die vorgelegte Arbeit weder im Inland noch im Ausland in gleicher oder ähnlicher Form einer anderen Prüfungsbehörde zum Zweck einer Promotion oder eines anderen Prüfungsverfahrens vorgelegt wurde. Alles aus anderen Quellen und von anderen Personen übernommene Material, das in der Arbeit verwendet wurde oder auf das direkt Bezug genommen wird, wurde als solches kenntlich gemacht. Insbesondere wurden alle Personen genannt, die direkt an der Entstehung der vorliegenden Arbeit beteiligt waren. Die aktuellen gesetzlichen Vorgaben in Bezug auf die Zulassung der klinischen Studien, die Bestimmungen des Tierschutzgesetzes, die Bestimmungen des Gentechnikgesetzes und die allgemeinen Datenschutzbestimmungen wurden eingehalten. Ich versichere, dass ich die Regelungen der Satzung der Universität Leipzig zur Sicherung guter wissenschaftlicher Praxis kenne und eingehalten habe.

Datum

Unterschrift 


\section{Erklärung über den Eigenanteil an der vorliegenden Arbeit}

Zunächst wurde gemeinsam mit den u. g. Betreuern eine Projektskizze entworfen.

Im Anschluss daran erfolgte die Datenerhebung in der Branddirektion Leipzig. Diese wurde im überwiegenden Teil eigenständig durchgeführt. Beratende Unterstützung erhielt ich durch Herrn Dr. med. R. Schröder sowie durch Frau Dr. A. Ramshorn-Zimmer. Bei der Auswertung der Protokolle wurde ich zudem durch den wissenschaftlichen Mitarbeiter Herrn M. Jäckel unterstützt.

Die statistische Auswertung erfolgte nach Beratung (Frau Dr. rer. nat. Christine Porzelius) durch das IMISE Leipzig (Institut für medizinische Informatik, Statistik und Epidemiologie) und Teilnahme am „Kurs zur Auswertung medizinischer Daten unter Verwendung des Statistikprogrammes SPSS" vom 19.05.-22.05.2014 eigenständig.

Die Erstellung der wissenschaftlichen Originalpublikation erfolgte eigenständig unter beratender Korrektur durch die u. g. Betreuer. Nach Einreichung beim Verlag und externer Begutachtung wurde die Publikation nach nochmaliger selbstständiger Überarbeitung veröffentlicht.

Katja Bader

Dr. med. A. Ramshorn-Zimmer

PD Dr. med. M. Bernhard

Prof. Dr. med. A. Gries 


\section{Lebenslauf}

\section{Persönliche Daten}

Name:

Katja Bader, geb. Jung

Geburtsdaten:

19.06.1985 in Leipzig

Familienstand:

verheiratet, 1 Kind

Adresse:

Davidstraße 9

04109 Leipzig

Telefon:

+49 160-96271432

Email:

mail@katjabader.de

\section{Schulausbildung}

09/1998-07/2004

Gymnasium Wilhelm Ostwald, Leipzig

vertieft mathematisch-naturwissenschaftliches Profil Abschluss Abitur

09/1996 - 07/1998

Gymnasium Neue Nikolaischule, Leipzig

09/1992 - 07/1996

Grundschule Heinrich-Mann, Leipzig

\section{Studium}

$10 / 2004-11 / 2010$

Humanmedizin, Universität Leipzig

Abschluss Staatsexamen

\section{Promotion}

Seit 09/2013

Entwicklung bodengebundener Notarzteinsätze im Stadtgebiet Leipzig von 2003 bis 2013 


\section{Berufstätigkeit}

Seit 05/2017

12/2013-09/2015

$11 / 2013$

Seit 09/2013

02/2012-08/2013

$01 / 2011-01 / 2012$

\section{Fortbildungskurse}

08/2016

09/2014

$03 / 2013$

$09 / 2012$

$02 / 2012$

\section{Praktika}

$04-06 / 2010$

$02-04 / 2010$

$12 / 2009-02 / 2010$

$08-12 / 2009$

\section{Elternzeit}

regelmäßige Teilnahme am Notarztdienst der Stadt Leipzig und der Stadt Leisnig

Zusatzbezeichnung Notfallmedizin

Zentrale Notaufnahme Universitätsklinikum Leipzig

Klinik für Innere Medizin, HELIOS Klinik Leisnig

Klinik für Herzchirurgie, Herzzentrum Leipzig
Grundkurs Echokardiographie Prof. Hagendorff, Leipzig

Grundkurs im Strahlenschutz

Kompaktkurs Notfallmedizin der SLÄK

Grundkurs interdisziplinäre Ultraschalldiagnostik der Landesärztekammer Thüringen

Einführungskurs Intensivmedizin der Arbeitsgemeinschaft Intensivmedizin, Arnsberg

Praktisches Jahr, Allgemeinmedizin, Praxis Dr. med. M. Burgkhardt

Praktisches Jahr, Innere Medizin,

General Hospital St. George's, Grenada

Praktisches Jahr, Gastroenterologie und Diabetologie,

HELIOS Klinik Borna

Praktisches Jahr, Klinik für Allgemein-, Visceral-, MIC- und Gefäßchirurgie, HELIOS Klinik Borna

\section{Sprachkenntnisse}

Englisch - fließend in Wort und Schrift 


\section{Vorstellung auf wissenschaftlichen Kongressen}

17.-19.06.2015

Posterpräsentation bei der Jahrestagung der Deutschen Gesellschaft für Internistische Intensiv- und Notfallmedizin (DGIIN), Köln (K. Bader)

„Entwicklung bodengebundener Notarzteinsätze

der Stadt Leipzig von 2003 bis 2013“

K. Jung ${ }^{1}$, A. Ramshorn-Zimmer ${ }^{1}$, M. Bernhard ${ }^{1}$, R. Schröder ${ }^{2}$, M. Kaul $^{2}$, A. Gries ${ }^{1}$

${ }^{1}$ Zentrale Notaufnahme, Universitätsklinikum Leipzig;

${ }^{2}$ Branddirektion, Stadt Leipzig 


\section{Danksagung}

Hiermit möchte ich mich bei Herrn Professor Dr. André Gries für die Überlassung des Themas und die langjährige Betreuung bedanken.

Danken möchte ich Frau Dr. Alexandra Ramshorn-Zimmer für die freundschaftliche Betreuung, die exzellente Organisation der gesamten Arbeit und die Möglichkeit jederzeit auftretende Fragen zu klären.

Ein besonderer Dank gilt Herrn Privatdozent Dr. med. habil. Michael Bernhard für die herausragende Hilfsbereitschaft und die unermüdliche Bereitschaft jederzeit Korrektur- und Verbesserungsvorschläge zu unterbreiten.

Mein Dank gilt auch Herrn Dr. Ralph Schröder und Herrn Kaul für die Bereitstellung der notwendigen Daten und die zur Verfügung Stellung des Arbeitsplatzes in der Branddirektion.

Zu guter Letzt gilt mein außerordentlicher Dank meiner Familie und meinen Freunden, auf deren Unterstützung ich stets in besonderem Maße zählen kann. 